\title{
The whole-genome and expression profile analysis of WRKY and RGAs in Dactylis glomerata showed that DG6C02319.1 and DgWRKYs may cooperate in the immunity against rust
}

\author{
Juncai Ren ${ }^{\text {Equal first author, } 1}{ }^{1}$, Jialing Hu ${ }^{\text {Equal first author, }{ }^{2} \text {, Ailing Zhang }}{ }^{2}$, Shuping Ren ${ }^{1}$, Tingting Jing ${ }^{2}$, Xiaoshan Wang ${ }^{2}$, Min \\ Sun ${ }^{2}$, Linkai Huang \\ Bing Zeng \\ ${ }^{1}$ College of Animal Science and Technology, Southwest University, Chongqing, Chongqing, China \\ 2 College of Grassland Science and Technology, Sichuan Agricultural University, Chengdu, Sichuan, China \\ Corresponding Authors: Linkai Huang, Bing Zeng \\ Email address: huanglinkai@sicau.edu.cn, zbin78@163.com
}

Orchardgrass (Dactylis glomerata) is one of the top four perennial forages worldwide and, despite its large economic advantages, often threatened by various environmental stresses. WRKY transcription factors (TFs) can regulate a variety of plant processes, widely participate in plant responses to biotic and abiotic stresses, and are one of the largest gene families in plants. WRKYs can usually bind W-box elements specifically. In this study, we identified a total of 93 DgWRKY genes and 281 RGAs, including 65, 169, and 47 nucleotide-binding site-leucine-rich repeats (NBS-LRRs), leucine-rich repeats receptor-like protein kinases (LRR-RLKs), and leucine-rich repeats receptor-like proteins (LRR-RLPs), respectively. Through analyzing the expression of $D g W R K Y$ genes in orchardgrass under different environmental stresses, it was found that many DgWRKY genes were differentially expressed under heat, drought, submergence, and rust stress. In particular, it was found that the greatest number of genes were differentially expressed under rust infection. Consistently, GO and KEGG enrichment analysis of all genes showed that 78 DgWRKY TFs were identified in the plant-pathogen interaction pathway, with 59 of them differentially expressed. Through cis-acting element prediction, 154 RGAs were found to contain W-box elements. Among them, DG6C02319.1 (a member of the LRR-RLK family) was identified as likely to interact with 14 DGWRKYs. Moreover, their expression levels in susceptible plants after rust inoculation were first up-regulated and then down-regulated, while those in the resistant plants were always up-regulated. In general, DgWRKYS responded to both biotic stress and abiotic stress. DgWRKYs and RGAs may synergistically respond to the response of orchardgrass to rust. This study provides meaningful insight into the molecular mechanisms of WRKY proteins in orchardgrass. 
1

$5 \quad$ Huang $^{2+}$, Bing Zeng ${ }^{1+}$

6

${ }^{+}$Co-Corresponding author

Corresponding Author:

Bing Zeng ${ }^{1}$

Linkai Huang ${ }^{2}$

\section{The whole-genome and expression profile analysis of WRKY and RGAs in Dactylis} glomerata showed that DG6C02319.1 and DgWRKYs may cooperate in the immunity against rust

Juncai Ren ${ }^{1 *}$, Jialing $\mathrm{Hu}^{2 *}$, Ailing Zhang ${ }^{2}$, Shuping Ren ${ }^{1}$, Tingting Jing ${ }^{2}$, Xiaoshan Wang ${ }^{2}$, Min Sun², Linkai

${ }^{1}$ College of Animal Science and Technology, Southwest University, Chongqing, China

${ }^{2}$ Department of Grassland Science, Faculty of Animal Science and Technology, Sichuan Agricultural

University, Chengdu, Sichuan, China

* these authors contributed equally to this work

Southwest University, Chongqing 402460, China

Email address: zbin78@163.com

Sichuan Agricultural University, Chengdu, Sichuan, 611130, China

Email address: huanglinkai@sicau.edu.cn

Abstract: Orchardgrass (Dactylis glomerata) is one of the top four perennial forages worldwide and, despite its

9 large economic advantages, often threatened by various environmental stresses. WRKY transcription factors

(TFs) can regulate a variety of plant processes, widely participate in plant responses to biotic and abiotic stresses, 
and are one of the largest gene families in plants. WRKYs can usually bind W-box elements specifically. In this study, we identified a total of $93 D g W R K Y$ genes and $281 R G A s$, including 65,169 , and 47 nucleotide-binding site-leucine-rich repeats (NBS-LRRs), leucine-rich repeats receptor-like protein kinases (LRR-RLKs), and leucine-rich repeats receptor-like proteins (LRR-RLPs), respectively. Through analyzing the expression of $D g W R K Y$ genes in orchardgrass under different environmental stresses, it was found that many $D g W R K Y$ genes were differentially expressed under heat, drought, submergence, and rust stress. In particular, it was found that the greatest number of genes were differentially expressed under rust infection. Consistently, GO and KEGG enrichment analysis of all genes showed that 78 DgWRKY TFs were identified in the plant-pathogen interaction pathway, with 59 of them differentially expressed. Through cis-acting element prediction, $154 R G A s$ were found to contain W-box elements. Among them, DG6C02319.1 (a member of the LRR-RLK family) was identified as likely to interact with 14 DGWRKYs. Moreover, their expression levels in susceptible plants after rust inoculation were first up-regulated and then down-regulated, while those in the resistant plants were always upregulated. In general, $D g W R K Y \mathrm{~s}$ responded to both biotic stress and abiotic stress. $D g W R K Y_{\mathrm{s}}$ and $R G A \mathrm{~s}$ may synergistically respond to the response of orchardgrass to rust. This study provides meaningful insight into the molecular mechanisms of WRKY proteins in orchardgrass.

Keywords: Dactylis glomerate; WRKY family; RGA family, abiotic stress; rust stress; transcriptome

\section{Introduction}

Animal husbandry is one of the most important industries involved in feeding humans, and it can provide humans with a higher quality of life. The processing, production, and quality of forage grass directly influence the output of livestock production. Currently, many countries, especially developed countries, pay much attention to raising and studying forage grass because of its crucial roles in the economy as well as in nutrition. It has been estimated that forage grass occupies $26 \%$ of land area and $70 \%$ of agricultural land (Conant 2010).

Orchardgrass (Dactylis glomerata L.), which belongs to the family Poaceae, is a cool-season forage grass planted worldwide (Hirata et al. 2011; Xie et al. 2014). As one of the top four perennial forage grasses from an economic perspective, orchardgrass is of vital importance in the production of meat from livestock and dairy production in temperate regions of the world (Wilkins \& Humphreys 2003). Furthermore, this species of grass possesses 
47

many advantages that have promoted its large-scale cultivation in America for grazing and hay harvest, including its fast growth, high yield, high sugar content, and shade tolerance (Tronsmo 1993; Volaire et al. 2001; Volaire 2003). Additionally, it is also used to establish grasslands in some places (Brummer \& Moore 2000). Orchardgrass has a wide adaptative range, but pathogens and some abiotic stresses considerably affect its quality and yield. Orchardgrass is susceptible to infection by pathogens such as rust fungus, which has caused substantial damage to populations of orchardgrass in natural grasslands, resulting in an approximately $69 \%$ increase in yellow and brown leaves within rust-infected areas, as shown by Pfender \& Alderman (2006). Similarly, Lancashire \& Latch (2012) found that rust stress reduces the tiller number and biomass of orchardgrass. Abiotic stresses resulting from global climate change, such as drought, heat, and waterlogging, also threaten the growth and quality of orchardgrass (Huang et al. 2014; Huang et al. 2015; Majidi et al. 2014; Zandalinas et al. 2018). Accordingly, improving the tolerance of orchardgrass to biotic and abiotic stresses is essential, and a very important step in achieving this goal is mining genes associated with resistance.

WRKY is one of the most important gene families in plants and was named based on its conserved DNA binding domain sequence, WRKYGQK. This conserved domain is approximately 60 residues followed by a $\mathrm{C}_{2} \mathrm{H}_{2}$ (Cys2His2) or $\mathrm{C}_{2} \mathrm{HC}$ (Cys2HisCys) zinc-binding motif (Eulgem et al. 2000; Rushton et al. 1996). The WRKY family can be divided into three groups (I-III) according to the number of domains and the zinc finger structure. Group II WRKY proteins can be classified into a, b, c, d, and e subgroups based on their primary amino acid sequences (Wu et al. 2019; Yan et al. 2019; Zhang \& Wang 2005).

WRKY transcription factor (TF) family were found to have an important relationship with abiotic tolerance and plant growth in Teak (Tectona Grandis) (Wang et al. 2020a), Camellia japonica (Yang et al. 2020), Eucalyptus globulus (Aguayo et al. 2019), sesame (Sesamum indicum L.) (Wang et al. 2021), peony (Paeonia lactiflora) (Wang et al. 2019), cassava (Manihot esculenta Crantz) (Wei et al. 2019) and so on. In cassava, mewrky20 can be activated by mehsp90.9 directly to encode a key enzyme involved in abscisic acid biosynthesis, and mewrky20-silenced plants displayed drought sensitivity, indicating its importance to the drought stress response (Wei et al. 2019). In pepper, overexpression of CaWRKY40 can enhance resistance to Ralstonia solanacearum and tolerance to heat stress of tobacco (Nicotiana tabacum L), while silencing of CaWRKY40 can enhance 
sensitivity to $R$. solanacearum and impair thermotolerance. Plwrky 70 from P. lactiflora belongs to the group III WRKY family, which was considerably suppressed under waterlogging treatment, dramatically dropping to minimum levels after $2 \mathrm{~h}$. This suggested that plwrky 70 was sensitive to waterlogging stresses in $P$. lactiflora (Han et al. 2019). Other than that, WRKY proteins could play an important role in resistance to pathogen attack in wild potato (Solanum commersonii and S. chacoense), through their involvement in specific signaling pathways (Villano et al. 2020). Researchers isolated WRKY genes in rice (Oryza sativa L.) infected by the fungal pathogen Magnaporthe grisea, and 15 of 45 genes showed remarkably increased expression under infection. WRKY W109669 was able to induce tobacco endo-1,3- $\beta$-glucanase (NtPR2) and promote systemic defense responses to tobacco mosaic virus in transgenic tobacco plants (Naoumkina et al. 2008). These results collectively indicate that many $W R K Y$ genes are very crucial to plant growth and plant resistance to abiotic stresses. Moreover, WRKY can participate in the process of plant defense against biological stress by regulating the expression of resistance genes ( $R$ genes) through several pathways.

Plants have complex pathogen recognition and defense mechanisms, including pathogen-associated molecular pattern-triggered immunity (PTI) and effect-triggered immunity (ETI), and $R$ genes, play an important role in the process of disease resistance (Zhang et al. 2014). $R$ genes have some common characteristics, and we can more broadly refer to resistance gene analogs $(R G A s)$, which are genes with the structural characteristics of $R$ genes. $R G A \mathrm{~s}$ can be divided into nucleotide-binding site-leucine-rich repeats (NBS-LRRs) and transmembrane leucine-rich repeats (TM-LRRs) (Sekhwal et al. 2015). TM-LRRs can be further subdivided into two categories: leucine-rich repeats receptor-like protein kinases (LRR-RLKs) and leucine-rich repeats receptor-like proteins (LRR-RLPs) (Hammond-Kosack \& Jones 1997).

To elucidate the roles of WRKYs in different species and improve the current understanding of biotic and abiotic stress responses at the molecular level, the identification and analysis of WRKY family members in target plant species is essential. Studies on the WRKY TF family in rice (Ross et al. 2007; Ryu et al. 2006), Glycyrrhiza glabra (Goyal et al. 2020), Rosa chinensis (Liu et al. 2019), and Saccharum spontaneum (Li et al. 2019) have already been conducted. These studies reveal that the WRKY TF family is crucially involved in biotic and abiotic stress responses and that some of these genes have been produced by duplication events. However, there is still 
99

no published research on the WRKY family in orchardgrass. As such, this study aimed to identify and analyze the WRKY TF family in orchardgrass to provide a foundation for future molecular genetic improvement. Our research team has previously created high-quality expression profile data of orchardgrass under drought, heat, and submergence treatments (Huang et al. 2015; Ji et al. 2018; Zeng et al. 2020). These data enable clarification of the mechanism by which WRKY TFs function in D. glomerata. By analyzing these data, we found that most $W R K Y$ genes, relative to control conditions, were differentially expressed under biological and abiotic stresses in orchardgrass, especially under rust stress, with $80 \%$ of $W R K Y$ genes showing changes in expression level. To further analyze the regulatory mechanism by which $D g W R K Y S$ respond to rust, we identified all $R G A s$ and their cis-acting elements and performed a weighted gene co-expression network analysis (WGCNA), revealing that $D g W R K Y$ s and $R G A$ s were highly likely to interact.

\section{Materials \& Methods}

\section{Sequence retrieval}

The published orchardgrass genome and protein sequences were downloaded from the orchardgrass genome database (http://orchardgrassgenome.sicau.edu.cn/download.php). The orchardgrass genome has been deposited under BioProject accession number PRJNA471014. The whole-genome assembly is composed of an approximately 1.84-Gb chromosome-scale diploid orchardgrass genome, including 40,088 protein-coding genes (Huang et al. 2020). The WRKY sequence data from A. thaliana (Araport11) were obtained from TAIR (https://www.arabidopsis.org/), while data from Triticum aestivum (using IWGSC(v2.2) gene annotation) were obtained from PlantTFDB (http://planttfdb.gao-lab.org/).

\section{Identification of WRKY proteins from orchardgrass}

We used two strategies to identify $W R K Y$ genes in D. glomerata. The first used HMMER SEARCH, in which we utilized HMMER v3 software (http://hmmer.janelia.org) to build an orchardgrass protein dataset. The Hidden Markov Model (HMM) file for WRKY (PF03106) domains was downloaded from Pfam (http://pfam.xfam.org/) (Finn et al. 2016) in order to identify WRKY proteins from the local database. The identification method (Lozano et al. 2015) was used to identify proteins using the raw WRKY HMM. A high-quality protein set (obtained using

PeerJ reviewing PDF | (2021:02:57835:2:0:NEW 19 Jun 2021) 
124

125

126

127

an E-value $<1 \times 10^{-20}$ and manual verification of intact WRKY domains) was aligned and then used to construct an orchardgrass-specific WRKY HMM using hmmbuild from the HMMER v3 suite. Next, we used the new orchardgrass-specific HMM to scan the protein data, and all proteins with an E-value lower than 0.01 were selected.

The second method utilized BLASTP. First, we selected T. aestivum and O. sativa WRKYs (as shown in Table $\mathrm{S} 1$; all sequences were downloaded from NCBI https://www.ncbi.nlm.nih.gov/) as the query sequences for a BLAST search of the protein sequences of D. glomerata. The sequences with an E-value less than $1 \mathrm{e}^{-10}$ were selected for further analysis. Finally, all DgWRKY sequences were verified using the online tool Search Pfam (http://pfam.xfam.org/search/), while sequences without a WRKY domain were removed. The selected protein sequences are shown in Table S2.

\section{Phylogenetic analysis and multiple sequence alignment}

Phylogenetic trees of genes from T. aestivum, D. glomerata, and A. thaliana were constructed with MEGA X utilizing the maximum likelihood method with a Poisson correction model and 1000 bootstrap replicates (Kumar et al. 2018). DNAMAN9 was used to analyze the core sequence of the WRKY domain from each subgroup of $93 \mathrm{DgWRKYs}$ after multiple sequence alignment.

\section{Chromosomal locations, motif analysis, and gene structure of DgWRKYs}

Chromosomal mapping was conducted using the MG2C online tool (http://mg2c.iask.in/mg2c_v2.1/). MEME (http://meme-suite.org/tools/meme/) was used to analyze motifs; the site distribution was any number of repetitions (ANR), and 10 consensus motifs were selected. Finally, the motifs and gene structure of DgWRKYs were mapped using TBtools. The coding sequence (CDS) of 93 orchardgrass genes were obtained from the orchardgrass genome data.

\section{Protein physical and chemical properties analysis and subcellular localization prediction}

The physicochemical properties of DgWRKYs were analyzed using ProtParam (https://web.expasy.org/protparam/), and estimates of the amino acid length, molecular weight (MW), theoretical 
148

149

isoelectric point (pI), instability index, aliphatic index, and grand average of hydropathicity (GRAVY) were obtained. Subcellular localization was predicted using WoLF PSORT (https://wolfpsort.hgc.jp/).

\section{Gene duplications and $K_{\mathrm{a}} / K_{\mathrm{s}}$ calculation}

McscanX was used to perform a collinear analysis of the orchardgrass genome (Wang et al. 2012). BLASTN was used to perform homologous CDS sequence comparison of WRKY family members. Based on previous research, gene duplication was constrained to gene pairs with lengths of aligned CDSs greater than $75 \%$ of the longer sequence with a similarity of the aligned region greater than $75 \%$ (Gu et al. 2002). Then, if a pair of duplicates was on the same chromosome and there are fewer than five genes between the two given genes, they were considered tandem duplicates; otherwise, the genes were considered to be segmented repeats (Cheng et al. 2018).

A circos diagram was drawn using circos software (http://circos.ca/). KaKs_Calculator2.0 was used to calculate the nonsynonymous substitution rate, $K_{\mathrm{a}}$, and the synonymous substitution rate, $K_{\mathrm{s}}$, of each duplicate gene pair.

\section{Expression profile analysis of $W R K Y$ genes under abiotic stresses and across different tissues}

The expression pattern data of $D g W R K Y$ genes under different abiotic stresses and in varied tissues has been previously measured by our research team (Huang et al. 2020; Huang et al. 2015; Ji et al. 2018; Zeng et al. 2020). This research has included expression profiles of orchardgrass under heat, drought, and submergence stress published by Huang et al. (2015), Ji et al. (2018), and Zeng et al. (2020), respectively. However, genome data for this species was not published until 2020, which required this previous work to be performed as unreferenced transcriptome analyses. In the present study, the raw data for heat and drought stress were re-downloaded in order to perform a reference analysis, and the expression profiles of $D g W R K Y \mathrm{~s}$ under different abiotic stresses were thus obtained. All fragments per kilobase of exon per million reads mapped (FPKM) estimated under stress are shown in Table S3.

\section{Expression profile analysis of $W R K Y$ genes under rust stress}

Two types of orchardgrass genetic lines, highly resistant PI251814 and highly susceptible PI292589 lines, were

Peer) reviewing PDF | (2021:02:57835:2:0:NEW 19 Jun 2021) 
172

173

174

175

176

177

used in this study. Four pots of the above two lines were cultivated, including two pots with highly resistant plants and two pots with highly susceptible plants. After a set cultivation period (at $20 \pm 5^{\circ} \mathrm{C}$ ), one pot containing highly resistant plants and one pot containing highly susceptible plants were inoculated (treatment group), respectively, while the other two pots received no treatment (control group). We used smearing to inoculate the plants with rust fungus. The spore pile was picked from the grass experiment base of the Ya'an campus of Sichuan Agricultural University.

The inoculated plants were placed in an incubator for dark treatment for $24 \mathrm{~h}\left(12^{\circ} \mathrm{C}\right.$, total darkness, $100 \%$ relative humidity) and then cultured for 14 days with 16 -h days $\left(20 \pm 2^{\circ} \mathrm{C}, 100 \%\right.$ relative humidity) and 8 -h nights (15 \pm $2{ }^{\circ} \mathrm{C}$, total darkness, $100 \%$ relative humidity). Latent spots appeared but were not obvious by 4 days after inoculation, while spore piles appeared by 7 days after inoculation. By 14 days after inoculation, the spore piles were fully mature, and the inoculated leaves showed symptoms of withering. The leaves of all plants were sampled on the 7th and 14th days for RNA-seq analysis (with two to three replicates per sample). A total of 24 samples were sent to Tianjin Novogene Co., Ltd. for RNA sequencing.

\section{Transcriptome analysis}

Bowtie V2.2.3 was utilized to establish a reference genome index (Langmead \& Salzberg 2012) based on the latest orchardgrass genome data published by our group (Huang et al. 2020). The double-ended clean-read sequences were compared with the reference genome using TopHat V2.0.12 (Kim et al. 2013). Then, the number of reads relative to each gene was calculated using HTSeq V0.6.1 (Anders et al. 2015), and the FPKM or TPM value of each gene was calculated according to the length of the gene and the number of reads per gene. If the expression level ratio between the experimental group and the control group was greater than 1.5 or less than $1.5^{-1}$, we considered that there was a differential expression (Data with zero or infinite ratios are deleted).

\section{GO and KEGG enrichment analysis}

Gene Ontology (GO) and Kyoto Encyclopedia of Genes and Genomes (KEGG) enrichment analyses of DgWRKYs were performed using OmicShare tools (https://www.omicshare.com/tools/).

\section{Identification of RGAs and analysis of cis-acting elements}

Identification of NBS-LRRs

PeerJ reviewing PDF | (2021:02:57835:2:0:NEW 19 Jun 2021) 
198

199

200

201

202

203

204

205

206

207

208

209

210

211

212

213

214

215

216

217

218

219

220

221

222

The IDs of the members of the NBS-LRR family were obtained from the NBS gene family articles published by our research group (Ren et al. 2020).

Identification of LRR-RLPs

A. thaliana RLP sequences were downloaded in order to BLASTP LRR-RLP family sequences. PFAM domain search (http://pfam.xfam.org/search\#tabview=tab1) was used to search for a sequence structure that does not contain the protein kinase (PKinase) domain structure but does contain the LRR structure domain sequence. TMHMM-2.0 (https://services.healthtech.dtu.dk/service.php?TMHMM-2.0) was used to identify whether the $\begin{array}{llllll}\text { sequences } & \text { contain } & \text { a } & \text { transmembrane } & \text { domain } & \text { structure. }\end{array}$ (https://services.healthtech.dtu.dk/service.php?SignalP-5.0) was used to identify the presence of a signal peptide. Identification of LRR-RLKs

RLK sequences were downloaded from NCBI, and BLASTP was performed using them. The genes containing LRR and PKinase domains were screened out using the PFAM database, and transmembrane domains and signaling proteins were searched for using TMHMM-2.0 and SignalP-5.0, respectively.

Prediction of cis-acting elements of $R G A$ genes

The orchardgrass genome sequence was downloaded from the orchardgrass genome database, and the 1.5-kb nucleic acid sequences upstream of each gene were extracted using TBtools. The identified sequences are shown in Table S4. Then, PlantCARE (http://bioinformatics.psb.ugent.be/webtools/plantcare/html/) was used to predict cis-acting element.

\section{WGCNA and Pearson correlation coefficient}

Pearson correlation coefficients were determined and WGCNA was conducted between WRKY and RGAs expression levels based on the expression of orchardgrass under rust infection. If a correlation was greater than 0.8 or less than -0.8 and the weighted correlation coefficient was greater than 0.5 , we deemed that correlation to be a very strong correlation.

\section{Results}

Identification and chromosomal locations of WRKYs in orchardgrass 
223

224

225

226

227

228

229

230

231

232

233

234

235

236

237

238

239

240

241

242

243

244

245

246

247

A total of 93 protein sequences with a WRKY domain were identified by BLASTP and/or HMMER. These identified proteins are encoded by genes located on all seven chromosomes (Fig. 1), except for three unmapped genes, and most of them are distributed on chromosomes 5 and 6, which contain 24 and 18 genes, respectively. Chromosomes 2 and 7 contain the fewest WRKY genes, only seven each. Based on their chromosomal locations, these 90 mapped $D g W R K Y$ genes were named from $D g W R K Y 1$ to $D g W R K Y 90$, while the remaining 3 unmapped DgWRKY genes were named DgWRKY0-1, DgWRKY0-2, and DgWRKY0-3. Then, the online tool Search Pfam was used to further retrieve the conserved domains and the locations of all sequences (Table S5). This analysis revealed that in addition to a WRKY domain, DgWRKY86, 37, 39, 40, 5, and 11 also contain a Plant zinc cluster domain (PF10533), while DgWRKY84 and 6 contains a Rx N-terminal domain (PF18052), and DgWRKY6 contains a NB-ARC domain (PF00931). As DgWRKY6 contains both NB-ARC and WRKY disease-resistant domains, NB-ARC is the characteristic domain of the NBS family, we conducted an online BLASTP of DgWRKY6 and found that it is a homolog of RPM1, a disease-resistance gene from Triticum urartu, and is therefore likely to be involved in disease resistance in orchardgrass.

\section{Protein physical and chemical properties analysis and subcellular localization prediction}

All of these proteins were analyzed using the ProtParam tool, which estimated amino acid length, MW, pI, the instability index, the aliphatic index, and GRAVY for each sequence. Meanwhile, WoLF PSORT was utilized to predict protein subcellular localization (Table 1). There were 51 (54.84\%) protein sequences with pI estimates less than 7: 82 (88.17\%) were located in the nucleus, 7 in the chloroplast, 2 in the cytoplasm, 1 in mitochondria, and 1 in the peroxisome. Only five sequences were estimated to be stable (i.e., instability index $<40$ ). The chromosome locations, WRKY domains, zinc finger motifs, and gene lengths of each protein were also determined (Table S6).

\section{Phylogenetic analysis and multiple sequence alignment of $W R K Y$ genes}

A total of 335 WRKY genes from A. thaliana (71 AtWRKYs), T. aestivum (171 TaWRKYs), and D. glomerata (93 $D g W R K Y s$ ) were used to construct a phylogenetic tree (Fig. 2). Based on the number of WRKY domains and the zinc finger motif that the sequences contain, these WRKYS were classified into three groups (groups I-III)

Peer] reviewing PDF | (2021:02:57835:2:0:NEW 19 Jun 2021) 
248

249

250

251

252

253

254

255

256

257

258

259

260

261

262

263

264

265

266

267

268

269

270

271

272

273

(Eulgem et al. 2000). Group I contained 13 members with two WRKY domains each located on both the Nterminus and $\mathrm{C}$-terminus and two zinc finger motifs of the $\mathrm{C}_{2} \mathrm{H}_{2}\left(\mathrm{CX}_{4-5} \mathrm{CX}_{22-23} \mathrm{HX}\right.$ H) type (Fig. 3A-B). Among them, one conserved domain of DgWRKY30 was mutated from WRKYGQK to WRKYGKR (Fig. 3A). Furthermore, according to the tree obtained, group II was classified into four subgroups, group $\mathbb{I}$ a, group $\mathbb{I} b$, group $I \mathrm{c}$, and group $\amalg \mathrm{d}$ (Fig. 2), which possessed 11, 18, 18, and 3 members, respectively. Each member of group II had one WRKY domain and one zinc finger motif (Fig. 3C-F) of the $\mathrm{C}_{2} \mathrm{H}_{2}$ type. In group II b, DgWRKY55 carried an incomplete WRKY conserved sequence, and DgWRKY44, 48, 52, 53, 72, and 83 exhibit a WRKYGKK variant sequence. Except for DgWRKY89, the others in groups $\amalg$ a and II c contain the $\mathrm{CX}_{5} \mathrm{CX}_{23} \mathrm{HX}_{1} \mathrm{H}$ motif while proteins in groups $I \mathrm{~b}$ and $\mathrm{I} \mathrm{d}$ contain $\mathrm{CX}_{4} \mathrm{CX}_{23} \mathrm{HX}_{1} \mathrm{H}$ zinc finger motifs, except for DgWRKY38 and DgWRKY52. DgWRKY38 and DgWRKY52 had $\mathrm{CX}_{4} \mathrm{CX}_{22} \mathrm{HX}_{1} \mathrm{H}$ and $\mathrm{CX}_{4} \mathrm{GX}_{23} \mathrm{HX}_{1} \mathrm{H}$ zinc finger motifs, respectively (Fig. 2). Thirty sequences belong to group III, each with one WRKY domain and one zinc finger motif (Fig. 3G-H) of type $\mathrm{C}_{2} \mathrm{HC}\left(\mathrm{CX}_{7} \mathrm{CX}_{23-28} \mathrm{HX}_{1} \mathrm{C}\right)$; however, DgWRKY6 had a different zinc finger motif sequence, $\mathrm{CX}_{7} \mathrm{CX}_{23} \mathrm{HX}_{1} \mathrm{Y}$. Additionally, DgWRKY87 was identified to carry a WRKY domain, but we manually retrieved it and found that it lacked a WRKYGQK heptapeptide (Fig. 3G); accordingly, we defined it as a WRKY-like gene.

\section{Gene structures and consensus motifs of WRKYs in orchardgrass}

A phylogenetic tree containing DgWRKYs using the maximum likelihood method was constructed using MEGA X (Fig. 4A). For this, we analyzed the consensus motifs determined by MEME and TBtools. By setting retrieval parameters, the distributions of 10 types of motifs in DgWRKYs were determined (Fig. 4B), and the members from the same subgroup had similar conserved motifs. All proteins contained motif 1, with a conserved WRKY amino acid sequence, except for DgWRKY87 (motif logos shown in Figure S1). Meanwhile, apart from some members of group III (DgWRKY21, 20, 87, 88, 61, 46, 60, 62, 63, 34, 18, 78, 76), all others contained motif 2, which shares sequence identity with a zinc finger motif. Motif 3, representing the $\mathrm{C}_{2} \mathrm{H}_{2}$ zinc finger structure, is distributed across all groups but group III. Motif 4 is mainly distributed in groups I and II b, while motif 5 is mainly distributed in group I but also in some parts of group II c. Motif 6 is mainly distributed in groups II a

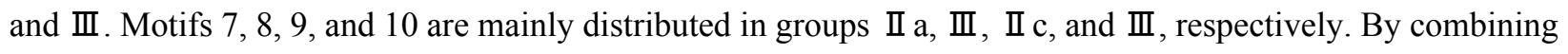


274 the analysis of motifs, it was determined that motifs 1,2 , and 3 represent part of the $\mathrm{C}_{2} \mathrm{H}_{2}$ structure while motifs 2751,2 , and 10 constituted part of the $\mathrm{C}_{2} \mathrm{HC}$ structure.

276 For further identification of the phylogenetic relationships among DgWRKYs, the position of the CDS and untranslated region (UTR) for each protein was determined (Fig. 4C). There were nine genes $(9.68 \%)$ that lacked an intron $(D g W R K Y 83,44,13,68,0-2,0-3,77,75,86)$, six of which (DgWRKY44, 68, 0-2, 0-3, 77, 75) had no UTR. Additionally, 14 genes (15.05\%) had two CDSs, 46 genes (49.46\%) had three CDSs, 16 genes (17.20\%) had four CDSs, 5 genes (5.37\%) had five CDSs, 3 genes (3.23\%) had six CDSs. Overall, DgWRKYs in the same subgroup had similar genetic structures throughout orchardgrass.

\section{Gene duplication and calculation of $K_{\mathrm{a}} / K_{\mathrm{s}}$}

Gene duplication is deemed to be one of the crucial drivers of the evolution of genomes and genetic systems. Segmental and tandem duplications are considered to be the two main phenomena underlying the expansion of plant gene families (Cannon et al. 2004). To study the duplication of WRKY genes throughout the evolution of orchardgrass, BLASTN and McscanX were used to perform a comparison of homologs, and 42 genes (45.16\%) were determined to be involved in duplication events, including 34 segmental duplicate genes (Fig. 5A) and 14 tandem duplicate genes (Some genes are both tandem duplicates and segmental duplicates). Six tandem duplicate gene pairs (DgWRKY20 \& DgWRKY21, DgWRKY26\& DgWRKY27, DgWRKY52 \& DgWRKY53, DgWRKY58 \& DgWRKY59, DgWRKY75 \& DgWRKY77, DgWRKY76 \& DgWRKY78) are shown in Fig. 5B to be distributed on chromosomes 3, 5, and 6. However, DgWRKY0-2 \& DgWRKY0-3 also comprise a pair of duplicate genes, though they cannot be localized to any chromosome in the published genome. The pairs DgWRKY26 \& $D g W R K Y 27$ and $D g W R K Y 0-2$ \& DGWRKY0-3 were identical duplicates, and each member of a pair shared the same amino acid sequence. The $K_{\mathrm{a}} / K_{\mathrm{s}}$ values of the four pairs of tandem duplicate genes were calculated (Table 2), and their values were low (i.e., $K_{\mathrm{a}} / K_{\mathrm{s}}<0.5$ ), which indicates that these genes have been subjected to purifying selection (Ziheng \& Rasmus 2000).

\section{Expression profile analysis of $W R K Y$ genes across orchardgrass tissues}

It has been reported that $W R K Y$ genes are expressed in a variety of cell types and under different physiological

Peer) reviewing PDF | (2021:02:57835:2:0:NEW 19 Jun 2021) 
299

300

301

302

303

304

305

conditions, enabling it to participate in the regulation of a variety of biological processes (Eulgem et al. 2000). In order to elucidate the expression pattern of $W R K Y \mathrm{~s}$ in different tissues of orchardgrass, a total of 72 expression profiles of $W R K Y$ genes in root, stem, leaf, flower, and spike tissues were obtained (Fig. 6). Most genes (44) were found to have the highest expression level in root samples, followed by spike samples (11 genes), while the leaf had the fewest genes with the highest observed expression level (4). Thus, it was obvious that WRKY genes are preferentially expressed in roots over leaves (Fig. 7). Notably, Ji et al. (2014) found that persistent drought damaged the leaves more than the roots in orchardgrass.

\section{Expression profile analysis of $W R K Y$ genes in orchardgrass under different abiotic stresses}

Members of the WRKY TF family are widely involved in the regulation of abiotic stresses in plants (Jiang et al. 2017). To further explore the potential functions of $D g W R K Y$ genes under various abiotic stresses, the expression patterns of $D g W R K Y$ genes under heat, drought, and submergence stress were determined. Thus, 60, 88, and 79 genes were found to be expressed under heat, drought, and submergence stress, respectively. After 10 days of heat stress, 19 genes were up-regulated. (Unless otherwise specified, the threshold for up-regulation and downregulation in this study is 1.5 -fold change, where the fold change is the ratio of FPKM or reads per kilobase per million mapped reads (RPKM) values). Additionally, 13 genes were down-regulated under heat treatment compared to the control in the heat-resistant 'BAOXING' cultivar (Fig. 8A). Additionally, three genes were discovered to be up-regulated by a more than 5-fold change, while DgWRKY73 was the most up-regulated (20fold). At the same time, 31 genes were down-regulated, and only one gene (DgWRKY41) was up-regulated in the heat-susceptible '01998' cultivar. After 26 days of heat stress, 9 and 16 genes were up-regulated and downregulated, respectively, in 'BAOXING,' while 5 and 26 genes were up-regulated and down-regulated, respectively, in '01998.' DgWRKY20 was up-regulated at 10 days and 26 days in 'BAOXING,' but downregulated in '01998.' Under heat stress, 22 genes only differentially expressed in 'BAOXING' after 10 days (Fig. 9A) and 12 genes only differentially expressed in 'BAOXING' after 26 days, while the express levels of none of these genes were changed in '01998' at the corresponding time (Fig. 9B).

The expression profiles of $W R K Y$ genes in leaf and root tissues from 'BAOXING' under drought stress were also assessed (Fig. 8B). After 18 days of drought treatment, 7 and 41 genes were up-regulated and 
325

326

327

328

329

330

331

332

333

334

335

336

337

338

339

340

341

342

343

344

345

346

347

348

349

350

downregulated, respectively, in leaf tissue, while 23 and 31 genes were up-regulated and down-regulated, respectively in root tissue. DgWRKY64 was upregulated, with 2-fold and 4-fold changes observed in leaf and root tissues, respectively. The expression levels of $D g W R K Y 18, D g W R K Y 49, D g W R K Y 51$, and DgWRKY64 were increased under both heat stress and drought stress. Additionally, 7 and 13 genes were up-regulated and downregulated, respectively, in root tissue only, but had no significantly changed in leaves (Fig. 9C).

The expression profiles of orchardgrass at 8 , and $24 \mathrm{~h}$ of submergence were constructed (Fig. 8C). Compared with the control group, 41 and 8 genes were up-regulated and down-regulated, respectively, in the submergencetolerant 'DIANBEI' cultivar at $8 \mathrm{~h}$ after submergence stress; among these, 12 up-regulated genes and 4 downregulated genes were only differentially expressed in 'DIANBEI' (Fig. 9D). By $24 \mathrm{~h}$ of the submergence stress treatment, 46 and 9 genes were up-regulated and down-regulated, respectively, in 'DIANBEI,' and 15 up- and 3 down-regulated genes were only differentially expressed in 'DIANBEI' (Fig. 9E). For the submergencesusceptible 'ANBA' cultivar, 40 and 6 genes were up-regulated and down-regulated after $8 \mathrm{~h}$ of submergence stress, while 39 and 10 genes were up-regulated and down-regulated after $24 \mathrm{~h}$ of submergence stress.

\section{Expression profile analysis of $W R K Y$ genes in orchardgrass under biotic stress}

WRKY TFs are also often involved in biotic stress responses (Jiang et al. 2017). To understand the WRKY genes involved in plant responses to biotic stress, we measured the expression levels of orchardgrass under rust infection and found 73 genes were related to rust stress (Fig. 10A). After 7 days of rust infection, 51 and 3 genes were up-regulated and down-regulated, respectively, in the highly rust-susceptible PI292589 line, while 53 and 5 genes were up-regulated and down-regulated in the highly rust-resistant PI251814 line. Additionally, 2 and 14 genes down-regulated and up-regulated, respectively, in PI251814 only (Fig. 9F). After 14 days of rust infection, 2 and 52 genes were up-regulated and down-regulated, respectively, in PI292589, while 47 genes and zero genes were up-regulated and down-regulated, respectively, in PI251814. Six of up-regulated genes exhibited varied expression levels only in PI251814 (Fig. 9G). The expression of most WRKY genes in susceptible and resistant plants were up-regulated on the 7th day of rust infection. However, as stress duration increased, only two genes were up-regulated in susceptible plants by the 14th day, while 47 genes were up-regulated in resistant plants. In order to prove the reliability of the data, some significance analyses were conducted on WRKY expression. As 
351

352

353

354

355

356

357

358

359

360

361

362

363

364

365

366

367

368

369

370

371

372

373

374

375

376

shown in Fig. 10B, there was a significant difference between PI251814 and PI292589. Significant difference existed between PI251814 before and after inoculation, but non-significant difference existed between PI292589 before and after inoculation (Fig 10C). There was no significant difference between high rust-resistant line and high rust-susceptible line before inoculation, but there was a significant difference after inoculation (Fig 10C). These results indicated that WRKY expression levels between PI251814 and PI292589 had little difference before inoculation with rust, but great changes occurred after inoculation with rust.

Notably, there were 53, 68, 70, and 73 differentially expressed genes (DEGs) at a 1.5-fold change threshold in the heat, drought, submergence, and rust stress treatments, respectively (Fig. 11). Relative to the control treatment, more DEGs were observed in the rust stress treatments than under other treatments. There were 37 common DEGs under all stresses, and 4 genes were differentially expressed only under rust stress (Fig. 9H).

\section{GO and KEGG enrichment analyses}

To further elucidate the biological function and molecular mechanisms of WRKY TFs, GO enrichment analysis and KEGG enrichment analysis of 93 genes were conducted. As seen in Fig. 12, the most genes were enriched for the terms membrane and membrane part among cell components; biological regulation, cellular process, metabolic process, and regulation among biological processes; and binding and nucleic acid binding TF activity among molecular functions. Unexpectedly, through KEGG enrichment analysis, we found that all the genes clustered into just three pathways: plant-pathogen interaction, mitogen-activated protein kinase (MAPK) signaling pathway-plant, and aminoacyl-tRNA biosynthesis (Fig. 13). It is well known that the first two pathways are generally associated with plant responses to environmental stress. Most were involved in plant-pathogen interaction, corresponding to 78 genes $(83.87 \%)$. Among them, 59 genes were differentially expressed under rust stress. This suggests that most WRKY TFs in orchardgrass are related to responses to pathogen interactions.

\section{Identification of $R G A s$ and their cis-acting elements}

According to the above results, $D g W R K Y s$ may be related to biotic stress responses, but how they participate in the defense process has been unclear. As we know, $R$ genes are usually involved in plant defense against pathogens (McHale et al. 2006). Therefore, investigating whether DgWRKYs can interact with $R G A$ s is of substantial importance. Using BLASTN and HMM SEARCH, 281 RGAs were identified, including 65 NBS-

Peer] reviewing PDF | (2021:02:57835:2:0:NEW 19 Jun 2021) 
377

378

379

380

381

382

383

384

385

386

387

388

389

390

391

392

393

394

395

396

397

398

399

400

401

402

LRRs, 169 LRR-RLKs, and 47 LRR-RLPs (RGAs protein sequences are shown in Table S7.). The NBS-LRR gene family was identified by Ren et al. (2020) in orchardgrass. W-box elements are the specific binding site of WRKYs (Eulgem et al. 2000). To determine whether WRKY TFs can participate in the response to rust stress by regulating the expression of $R G A s, 1.5-\mathrm{kb}$ nucleic acid sequences upstream of $R G A s$ were extracted for prediction of cis-acting elements. As shown in Fig. 14, 154 of the 218 RGAs have W-box elements, with DG3C03551.1 containing the most W-box elements (4).

\section{WGCNA and co-expression analyses of WRKYs with $R G A s$}

WGCNA and correlation analyses were also performed for WRKY and RGAs. As shown in Fig. 15, principal component analyses (PCAs) showed that the high-resistance plants 7 and 14 days after rust inoculation (HR_7 and HR_14, respectively) were quite distinct from other plants. This illustrates the difference between highresistance and high-sensitivity plants. A total of nine modules were identified by WGCNA. Among the modules shown in Fig. 16, the turquoise and brown ones were significantly correlated with HR_7 and HR_14, respectively. Therefore, we extracted the data associated with these two modules for further analysis, finding 1261 pairs of interactions, among which we removed low reliability interactions (weight $<0.5$ ), thus retaining the high reliability $W R K Y-R G A$ interactions. Additionally, the $R G A s$ without a W-box element were removed. Pearson correlation coefficients describing the relationship between the expression levels of each gene pair were calculated for verification, and a high-quality interaction map was finally obtained (Table 3). Ultimately, there were 24 interactions identified between 14 WRKYs and 5 RGAs (Fig. 17). It is worth noting that DG6C02319.1 (LRR-RLK family member) was determined to potentially interact with all $14 W R K Y_{s}$, for which we proposed several hypotheses. One possibility is that DG6C02319.1 can induce the expression of WRKYs after inoculation with rust fungus to resist rust fungus invasion. The second is that $D G 6 C 02319.1$ is not the only gene that can induce the expression of $W R K Y \mathrm{~s}$, as $W R K Y \mathrm{~s}$ still regulate the transcription of $D G 6 C 02319.1$ (because the promoter region of $D G 6 C 02319.1$ contains a W-box element). The third possibility is that various $W R K Y \mathrm{~s}$ coordinate and regulate the transcription of $D G 6 C 02319.1$ to participate in the immune process of plants against rust.

\section{Discussion}


403

404

405

406

407

408

409

410

411

412

413

414

415

416

417

418

419

420

421

422

423

424

425

426

427

428

429

Animal husbandry depends on the feed industry, but feed resources have become a major constraint on animal production in Asia (Devendra \& Sevilla 2002). Orchardgrass is one of the top four perennials forage crops globally. It plays an important role in the production of meat and dairy products (Wilkins \& Humphreys 2003), but its yield is threatened by various environmental stresses. A large number of studies have shown that WRKY TFs can regulate a variety of plant processes and responses to various biotic and abiotic stresses (Cai et al. 2021; Wang et al. 2020b; Wang et al. 2020c; Xiang et al. 2021). In this study, WRKY family members were first identified from orchardgrass, and all sequences were located, classified, and analyzed according to their expression profiles, which is of great significance for molecular-assisted breeding of orchardgrass.

A total of $93 D g W R K Y$ coding genes were identified and divided into three groups according to the number of WRKY domains and the type of zinc finger structure carried in the protein sequence. Group $\mathbb{I}$ had the most members, which was consistent with previous reports of WRKYs in A. thaliana, M. esculenta, G. max, and Caragana intermedia (Bencke-Malato et al. 2014; Eulgem et al. 2000; Wan et al. 2018; Wei et al. 2016). The WRKYGQK heptapeptide stretch is considered to be an important sequence for identifying and binding the Wbox element of the target gene promoter (Rushton et al. 2010). In orchardgrass, some TFs exhibit variants differing from the WRKYGQK heptapeptide stretch, including WRKYGEK, WRKYGKK, and WKKYGQK (Fig. 3), and certain TFs (i.e., DgWRKY55, WRKSYYR; Fig. 3) did not contain the full WRKY amino acid sequence or even had no WRKY (i.e., DgWRKY87, Fig. 3) tetrapeptide. As far as we know, WRKSYYR is a WRKY gene family variant unique to orchardgrass. Additionally, all six TFs (DgWRKY44, 48, 52, 53, 72, 83) exhibiting the WRKYGKK heptapeptide variant belong to group II b (Fig. 3). Research has shown that WRKY TFs with a variant heptapeptide may recognize binding elements outside W-box elements; this includes WRKYGKK, which can specifically bind with WK-box elements (TTTTCCAC) (van Verk et al. 2008; Zhou et al. 2008). Four TFs (DgWRKY18, 34, 76, 78) with the WRKYGEK heptapeptide variant belong to group III and are located in the nucleus, and they were upregulated during drought and/or submergence stresses. Moreover, the expression of $D g$ WRKY18 increased after treatments with each of the three abiotic stresses. The establishment of these variant sequences in WRKY gene family members correspond with long-term gene family evolution. WKKYGQK variants were distributed in groups $\mathbb{I} \mathrm{d}$ and III. At the same time, we also found that most duplicate WRKY genes in orchardgrass (90.48\%) belonged to groups $\mathbb{I I}$ and $\mathbb{I}$, and it is obvious from the tree in Fig. 2 
430

431

432

433

434

436

that members of group III are divided into two branches, which indicates that the WRKY genes of these two branches possibly originated from different ancestors. Overall, groups II and III were more diverse, consistent with the results of Zhang \& Wang (2005).

Studies have shown that the expression of genes with short introns or short total intron length are increased in plants (Chung et al. 2006). Among all WRKY genes identified in orchardgrass, nine genes had no introns. The expression levels of five of them in roots, stems, leaves, flowers, and spikes were measured. We found that three of these five genes (DgWRKY13, 44, 83) had higher FPKM values (Fig. 6).

A recent study by Mohanta et al. (2016) has suggested that WRKY TFs may also contain more than two WRKY domains or other domains, including ZF SBP, LRR, and PAH domains. We also found that some DgWRKY TFs possess some other conserved domains, for example, Plant zinc cluster, Rx N-terminal, NB-ARC domains (Table S5).

As is widely known, TFs are usually involved in transcriptional regulation in the nucleus (Brownawell et al. 2001), which is consistent with our results. Moreover, $88.7 \%$ of $\operatorname{DgWRKYS}$ are located in the nucleus (Table 1), and GO enrichment analysis has also showed that most TFs were enriched in the membrane, nucleic acid binding TF activity, and biological regulation categories (Fig. 12). Based on GO enrichment analysis, some WRKY TFs are located in chloroplasts, the cytoplasm, mitochondria, and peroxisomes, while some even may be involved in transcriptional regulation outside of the cytoplasm (Table 1).

$D g W R K Y s$ were induced by various abiotic stresses, including heat, drought, and waterlogging, and they are also expressed in roots, stems, leaves, flowers, and spikes of orchardgrass, with roots and leaves exhibiting the highest and lowest expression levels respectively (Fig. 7). Additionally, the number of DEGs in root tissue was more than that in leaf tissue (Fig. 8B). Ji et al. (2014) reported that under the same intensity of drought stress, the damage to leaves was much greater than that to roots. This result indicates that $D g W R K Y$ genes play an important role in the response to drought stress and root growth.

Rust stress also changed the expression of $D g W R K Y$ genes. By comparing the number of DEGs under different stresses, we found that, relative to control conditions, the most DEGs were revealed under rust stress (Fig. 11). 
455

456

457

458

459

460

461

462

463

464

465

466

467

468

469

470

471

472

473

474

475

476

477

478

479

480

Through KEGG enrichment analysis of 93 WRKY transcription factors, it was found that 78 transcription factors clustered in the plant-pathogen interaction pathway (Fig. 13), among which, 59 genes were differentially expressed under rust stress. These results indicate that $D g W R K Y s$ play an important role in plant responses to rust stress. Plant responses to pathogen attacks require large-scale transcriptional reprogramming, including transcriptional reprogramming by $W R K Y$ gene family members. Many $W R K Y$ genes can negatively regulate plant defense signaling, including AtWRKY7, 11, and 17, and mutations in these genes can induce susceptibility to virulent Pseudomonas syringae (Journot-Catalino et al. 2006; Pandey \& Somssich 2009). Positive regulation of plant disease resistance signaling WRKY genes has also been observed in CaWRKY27, TaWRKY70, and WRKY22, among other genes (Cheng \& Wang 2014; Jiang et al. 2017; Wang et al. 2017). Furthermore, overexpression of WRKY22 increased resistance to Pyricularia oryzae Cav. in rice. In orchardgrass, rust-resistant plants clearly showed the most $W R K Y$ genes that increased 7 and 14 days after rust inoculation (53 and 5 genes up-regulated and down-regulated, respectively at day 7 and 47 genes and zero genes up-regulated and downregulated, respectively, at day 14). However, in susceptible plants, after 7 days of rust stress, there were 51 genes up-regulated and just 3 genes downregulated. As the stress duration increased to 14 days, only two WRKY genes were up-regulated, while the expression of 52 genes was reduced (Fig. 10A). This suggests that most $D g W R K Y$ genes were more likely to act as positive regulators of plant disease resistance signals in orchardgrass. $\operatorname{DgWRKY9}, 13,14,50$, and 57 showed a tendency of first being up-regulated and then down-regulated in susceptible plants, but showed a tendency of consistent up-regulation in highly resistant plants. Accordingly, these genes could be further studied as candidate genes.

DG6C02319.1 is an $L R R-R L K$ gene, and all relevant results indicated that it is strongly co-expressed with 14 $W R K Y$ genes. It has been shown that $L R R-R L K$ can act as a pattern recognition receptor (PRR) to stimulate PAMP-triggered immunity (PTI) in a plant by recognizing the conserved PAMP structure (Boller \& Felix 2009; Dodds \& Rathjen 2010). FLS2 is a typical $L R R-R L K$ gene that has been confirmed encode a protein that regulates the complete MAPK signaling pathway, e.g., WRKY22/WRKY29, MKK4/MKK5, MPK3/MPK6, etc. (Thomma et al. 2011).

On A. thaliana chromosome 4, the promoters of many genes are rich in W-box elements, four of which encode

Peer] reviewing PDF | (2021:02:57835:2:0:NEW 19 Jun 2021) 
481

482

483

484

485

486

487

488

489

490

491

492

493

494

495

496

497

498

499

500

501

502

503

504

505

506

507

RLKs. Both salicylic acid (SA) treatment and Pseudomonas syringae infection resulted in increased expression levels of these genes. A gel retardation assay showed that the W-box of the $R L K 4$ promoter can be recognized by purified AtWRKY18 and SA-induced Arabidopsis nuclear extract. A further transgenic analysis shows that these W-box elements play important roles in inducing the expression of reporter genes (Du \& Chen 2000). RLK senses external stimuli and phosphorylates specific target proteins through its kinase activity, thereby transducing signals into cells (Czernic et al. 1999). These results suggest that WRKY may regulate genes encoding signal transduction proteins. The promoter regions of PR-1 and NPR1 contain W-boxes. The binding of WRKY transcription factors to PR-1 can quickly activate the signaling of early defense responses in plants, and the binding of WRKY transcription factors to NPR1 can in turn regulate NPR1 to coordinate the expression of $R$ genes ( $Y u$ et al. 2001). These results are consistent with our experimental results.

\section{Conclusions}

In total, $93 W R K Y$ genes were identified from the orchardgrass genome, and the structure of $D g W R K Y$ genes was thus revealed. $D g W R K Y 87$ is a WRKY-like gene with a structure similar to that of WRKY genes, but without the WRKYGQK heptapeptide. The physical and chemical properties and subcellular location of the identified proteins were predicted. Through the analysis of the expression profile of $D g W R K Y_{\mathrm{s}}$, it was found that most $D g W R K Y s$ showed differential expression under various stresses, including heat, drought, submergence, and rust stress. This indicates that $D g W R K Y$ genes are involved in a variety of environmental stresses and processes, including biotic and abiotic stresses. Relative to control conditions, 73 DEGs (accounting for $80 \%$ of all $D g W R K Y$ genes) were observed under rust stress, and through GO and KEGG annotations, 78 WRKY TFs were observed to be enriched in pathogen interaction pathways, suggesting that WRKY genes in orchardgrass play an important role in the antibacterial defense system. Through cis-acting element prediction, WGCNA, and coexpression analysis, five $R G A \mathrm{~s}$ and fourteen $W R K Y \mathrm{~s}$ with interactions were found. This work provides a firm foundation for further functional studies of WRKY TFs in plants.

\section{References}

Aguayo P, Lagos C, Conejera D, Medina D, and Valenzuela S. 2019. Transcriptome-wide identification of WRKY family genes and their expression under cold acclimation in Eucalyptus globulus. Trees, 33(10), 1-15. 10.1007/s00468-019-01860-3. 
508

509

510

511

512

513

514

515

516

517

518

519

520

521

522

523

524

525

526

527

528

529

530

531

532

533

534

535

536

537

538

539

540

541

542

543

544

545

546

547

Anders S, Pyl PT, and Huber W. 2015. HTSeq--a Python framework to work with high-throughput sequencing data. Bioinformatics 31:166-169. 10.1093/bioinformatics/btu638.

Bencke-Malato M, Cabreira C, Wiebke-Strohm B, Bücker-Neto L, and Mancini E. 2014. Genomewide annotation of the soybean WRKY family and functional characterization of genes involved in response to Phakopsora pachyrhiziinfection. BMC Plant Biology 14: 236.

Boller T, and Felix G. 2009. A renaissance of elicitors: perception of microbe-associated molecular patterns and danger signals by pattern-recognition receptors. Annu Rev Plant Biol 60:379406. 10.1146/annurev.arplant.57.032905.105346.

Brownawell AM, Kops GJ, Macara IG, and Burgering BM. 2001. Inhibition of nuclear import by protein kinase $\mathrm{B}(\mathrm{Akt})$ regulates the subcellular distribution and activity of the forkhead transcription factor AFX. Mol Cell Biol 21:3534-3546. 10.1128/MCB.21.10.35343546.2001.

Brummer EC, and Moore KJ. 2000. Persistence of perennial cool-season grass and legume cultivars under continuous grazing by beef cattle. Agronomy Journal 92:466-471.

Cai H, Huang Y, Chen F, Liu L, Chai M, Zhang M, Yan M, Aslam M, He Q, and Qin Y. 2021. ERECTA signaling regulates plant immune responses via chromatin - mediated promotion of WRKY33 binding to target genes. New Phytologist. 230. 10.1111/nph.17200.

Cannon SB, Mitra A, Baumgarten A, Young ND, and May G. 2004. The roles of segmental and tandem gene duplication in the evolution of large gene families in Arabidopsis thaliana. BMC Plant Biol 4:10. 10.1186/1471-2229-4-10.

Cheng H, and Wang S. 2014. WRKY-Type Transcription Factors: a Significant Factor in RicePathogen Interactions. SCIENTIA SINICA Vitae 44:784-793. 10.1360/052014-97.

Cheng X, Su X, Muhammad A, Li M, Zhang J, Sun Y, Li G, Jin Q, Cai Y, and Lin Y. 2018. Molecular Characterization, Evolution, and Expression Profiling of the Dirigent (DIR) Family Genes in Chinese White Pear (Pyrus bretschneideri). Front Genet 9:136. 10.3389/fgene.2018.00136.

Chung BY, Simons C, Firth AE, Brown CM, and Hellens RP. 2006. Effect of 5'UTR introns on gene expression in Arabidopsis thaliana. BMC Genomics 7:120. 10.1186/1471-2164-7120.

Conant RT. 2010. Challenges and opportunities for carbon sequestration in grassland systems. Integrated Crop Management 9.

Czernic P, Visser B, Sun W, Savouré A, Deslandes L, Marco Y, Van Montagu M, and Verbruggen N. 1999. Characterization of an Arabidopsis thaliana receptor-like protein kinase gene activated by oxidative stress and pathogen attack. 18:321-327. https://doi.org/10.1046/j.1365-313X.1999.00447.x.

Devendra C, and Sevilla CC. 2002. Availability and use of feed resources in crop-animal systems in Asia. Agricultural systems 71:59-73.

Dodds PN, and Rathjen JP. 2010. Plant immunity: towards an integrated view of plant-pathogen interactions. Nat Rev Genet 11:539-548. 10.1038/nrg2812.

Du L, and Chen Z. 2000. Identification of genes encoding receptor-like protein kinases as possible 
targets of pathogen- and salicylic acid-induced WRKY DNA-binding proteins in Arabidopsis. 24:837-847. 10.1111/j.1365-313X.2000.00923.x.

Eulgem T, Rushton PJ, Robatzek S, and Somssich IE. 2000. The WRKY superfamily of plant transcription factors. Trends in Plant Science 5:199-206.

Finn RD, Coggill P, Eberhardt RY, Eddy SR, Mistry J, Mitchell AL, Potter SC, Punta M, Qureshi M, Sangrador-Vegas A, Salazar GA, Tate J, and Bateman A. 2016. The Pfam protein families database: towards a more sustainable future. Nucleic Acids Res 44:D279-285. 10.1093/nar/gkv1344.

Goyal P, Muzafar M, Vishwakarma R, Sharma D, Dhar M K, and Gupta S. 2020. A Comprehensive Transcriptome-Wide Identification and Screening of WRKY Gene Family Engaged in Abiotic Stress in Glycyrrhiza glabra. Scientific Reports. 10. 10.1038/s41598019-57232-x.

Gu Z, Cavalcanti A, Chen FC, and Bouman P, \& Li, W. H. 2002. Extent of gene duplication in the genomes of Drosophila, nematode, and yeast. Molecular biology and evolution 19:256262.

Hammond-Kosack K, and Jones J. 1997. Plant disease resistance genes. Annu. Rev. Plant Phys. Annual review of plant physiology and plant molecular biology 48:575-607. 10.1146/annurev.arplant.48.1.575.

Han C, Li J, Ma Y, Guo J, Guo X, and Xu J. 2019. PIWRKY70: a Paeonia lactiflora transcription factor that sensitively responds to low-temperature, salt and waterlogging stresses. Canadian Journal of Plant Science.

Hirata M, Yuyama N, and Cai H. 2011. Isolation and characterization of simple sequence repeat markers for the tetraploid forage grass Dactylis glomerata. Plant Breeding 130:503-506. 10.1111/j.1439-0523.2010.01831.x.

Huang L, Feng G, Yan H, Zhang Z, Bushman BS, Wang J, Bombarely A, Li M, Yang Z, Nie G, Xie W, Xu L, Chen P, Zhao X, Jiang W, and Zhang X. 2020. Genome assembly provides insights into the genome evolution and flowering regulation of orchardgrass. Plant Biotechnol J 18:373-388. 10.1111/pbi.13205.

Huang L, Yan H, Jiang X, Zhang Y, Zhang X, Ji Y, Zeng B, Xu B, Yin G, Lee S, Yan Y, Ma X, and Peng Y. 2014. Reference gene selection for quantitative real-time reverse-transcriptase PCR in orchardgrass subjected to various abiotic stresses. Gene 553:158-165. 10.1016/j.gene.2014.10.017.

Huang LK, Yan HD, Zhao XX, Zhang XQ, Wang J, Frazier T, Yin G, Huang X, Yan DF, Zang WJ, Ma X, Peng Y, Yan YH, and Liu W. 2015. Identifying differentially expressed genes under heat stress and developing molecular markers in orchardgrass (Dactylis glomerata L.) through transcriptome analysis. Mol Ecol Resour 15:1497-1509. 10.1111/17550998.12418.

Ji Y, Chen P, Chen J, Pennerman KK, Liang X, Yan H, Zhou S, Feng G, Wang C, Yin G, Zhang X, Hu Y, and Huang L. 2018. Combinations of Small RNA, RNA, and Degradome Sequencing Uncovers the Expression Pattern of microRNA(-)mRNA Pairs Adapting to Drought Stress in Leaf and Root of Dactylis glomerata L. Int J Mol Sci 19. 
10.3390/ijms19103114.

Ji Y, Zhang X, Peng Y, Liang X, Huang L, Ma X, and Ma Y. 2014. Effects of drought stress on lipid peroxidation, osmotic adjustment and activities of protective enzymes in the roots and leaves of orchardgrass. Acta Prataculture Sin 23:144-151.

Jiang J, Ma S, Ye N, Jiang M, Cao J, and Zhang J. 2017. WRKY transcription factors in plant responses to stresses. J Integr Plant Biol 59:86-101. 10.1111/jipb.12513.

Journot-Catalino N, Somssich IE, Roby D, and Kroj T. 2006. The transcription factors WRKY11 and WRKY17 act as negative regulators of basal resistance in Arabidopsis thaliana. Plant Cell 18:3289-3302. 10.1105/tpc.106.044149.

Kim D, Pertea G, Trapnell C, Pimentel H, Kelley R, and Salzberg SL. 2013. TopHat2: accurate alignment of transcriptomes in the presence of insertions, deletions and gene fusions. Genome Biology 14:R36.

Kumar S, Stecher G, Li M, Knyaz C, and Tamura K. 2018. MEGA X: Molecular Evolutionary Genetics Analysis across Computing Platforms. Mol Biol Evol 35:1547-1549. 10.1093/molbev/msy096.

Lancashire JA, and Latch GCM. 2012. Some effects of stem rust (Puccinia graminisPers.) on the growth of cocksfoot (Dactylis glomerataL. 'Grasslands Apanui'). New Zealand Journal of Agricultural Research 12:697-702. 10.1080/00288233.1969.10421221.

Langmead B, and Salzberg SL. 2012. Fast gapped-read alignment with Bowtie 2. Nat Methods 9:357-359. 10.1038/nmeth.1923.

Li Z, Hua X, Zhong W, Yuan Y, Wang Y, Wang Z, Ming, and Zhang J. 2019. Genome-Wide Identification and Expression Profile Analysis of WRKY Family Genes in the Autopolyploid Saccharum spontaneum. Plant \& cell physiology. 61. 10.1093/pcp/pcz227.

Liu X, Li D, Zhang S, Xu Y, and Zhang Z. 2019. Genome-wide characterization of the rose (Rosa chinensis) WRKY family and role of RcWRKY41 in gray mold resistance. BMC Plant Biology. 19. 10.1186/s12870-019-2139-6.

Lozano R, Hamblin MT, Prochnik S, and Jannink JL. 2015. Identification and distribution of the NBS-LRR gene family in the Cassava genome. BMC Genomics 16:360. 10.1186/s12864015-1554-9.

Majidi MM, Hoseini B, Abtahi M, Mirlohi A, and Araghi B. 2014. Genetic analysis of seed related traits in Orchardgrass (Dactylis glomerata) under normal and drought stress conditions. Euphytica 203:409-420. 10.1007/s10681-014-1299-6.

McHale L, Tan X, Koehl P, and Michelmore RW. 2006. Plant NBS-LRR proteins: adaptable guards. Genome Biol 7:212. 10.1186/gb-2006-7-4-212.

Mohanta TK, Park YH, and Bae H. 2016. Novel Genomic and Evolutionary Insight of WRKY Transcription Factors in Plant Lineage. Sci Rep 6:37309. 10.1038/srep37309.

Naoumkina MA, He XZ, and Dixon RA. 2008. Elicitor-induced transcription factors for metabolic reprogramming of secondary metabolism in Medicago truncatula. BMC Plant Biology 8:132.

Pandey SP, and Somssich IE. 2009. The role of WRKY transcription factors in plant immunity. Plant Physiology 150:1648-1655. 10.1104/pp.109.138990. 
630 Pfender W F \& Alderman S C. 2006. Regional Development of Orchardgrass Choke and Estimation of Seed Yield Loss. Plant Disease 90:240-244.

Ren S, Sun M, Yan H, Wu B, Jing T, Huang L, and Zeng B. 2020. Identification and Distribution of NBS-Encoding Resistance Genes of Dactylis glomerata L. and Its Expression Under Abiotic and Biotic Stress. Biochem Genet. 10.1007/s 10528-020-09977-8.

Ross CA, Liu Y, and Shen QJ. 2007. The WRKY gene family in rice (Oryza sativa). Journal of Integrative Plant Biology 49:827-842.

Rushton PJ, Somssich IE, Ringler P, and Shen QJ. 2010. WRKY transcription factors. Trends Plant Sci 15:247-258. 10.1016/j.tplants.2010.02.006.

Rushton PJ, Torres JT, Parniske M, Wernert P, Hahlbrock K, and Somssich IE. 1996. Interaction of elicitor - induced DNA - binding proteins with elicitor response elements in the promoters of parsley PR1 genes. The EMBO Journal 15:5690-5700.

Ryu HS, Han M, Lee SK, Cho JI, Ryoo N, and Heu S. 2006. A comprehensive expression analysis of theWRKYgene superfamily in rice plants during defense response. Plant cell reports 25:836-847.

Sekhwal MK, Li P, Lam I, Wang X, Cloutier S, and You FM. 2015. Disease Resistance Gene Analogs (RGAs) in Plants. 16:19248-19290.

Thomma BPHJ, Nürnberger T, and Joosten MHAJ. 2011. Of PAMPs and Effectors: The Blurred PTI-ETI Dichotomy. The Plant Cell 23:4-15. 10.1105/tpc.110.082602.

Tronsmo AM. 1993. Resistance to Winter Stress Factors in Half-Sib Families ofDactylis glomerata, Tested in a Controlled Environment. Acta Agriculturae Scandinavica, Section B - Soil \& Plant Science 43:89-96. 10.1080/09064719309411224.

van Verk MC, Pappaioannou D, Neeleman L, Bol JF, and Linthorst HJ. 2008. A Novel WRKY transcription factor is required for induction of PR-1a gene expression by salicylic acid and bacterial elicitors. Plant Physiology 146:1983-1995. 10.1104/pp.107.112789.

Villano C, Esposito S, D'Amelia V, Garramone R, and Carputo D. 2020. WRKY genes family study reveals tissue-specific and stress-responsive tfs in wild potato species. Scientific Reports, 10(1). 10.1038/s41598-020-63823-w.

Volaire F. 2003. Seedling survival under drought differs between an annual (Hordeum vulgare) and a perennial grass (Dactylis glomerata). New Phytologist 160:501-510. 10.1046/j.14698137.2003.00906.x.

Volaire F, Conéjero G, and Lelièvre F. 2001. Drought survival and dehydration tolerance in Dactylis glomerata and Poa bulbosa. Functional Plant Biology 28:743-754. https://doi.org/10.1071/PP00162.

Wan Y, Mao M, Wan D, Yang Q, Yang F, Mandlaa, Li G, and Wang R. 2018. Identification of the WRKY gene family and functional analysis of two genes in Caragana intermedia. BMC Plant Biol 18:31. 10.1186/s12870-018-1235-3.

Wang H, Zou S, Li Y, Lin F, and Tang D. 2020b. An ankyrin-repeat and WRKY-domaincontaining immune receptor confers stripe rust resistance in wheat. Nature 
Communications. 11. 1353. 10.1038/s41467-020-15139-6.

Wang J, Tao F, An F, Zou Y, Tian W, Chen X, Xu X, and Hu X. 2017. Wheat transcription factor TaWRKY70 is positively involved in high-temperature seedling plant resistance to Puccinia striiformis f. sp. tritici. Mol Plant Pathol 18:649-661. 10.1111/mpp.12425.

Wang L, Dossa K, You J, Zhang Y, Li D, Zhou R, Yu J, Wei X, Zhu X, Jiang S, Gao Y, Ali Mmadi $M$ and Zhang X. 2020c. High-resolution temporal transcriptome sequencing unravels ERF and WRKY as the master players in the regulatory networks underlying sesame responses to waterlogging and recovery. Genomics. 113. 10.1016/j.ygeno.2020.11.022.

Wang X, Li J, Guo X, Ma Y, Qiao Q, and Guo J. 2019. PIWRKY13: A Transcription Factor Involved in Abiotic and Biotic Stress Responses in Paeonia lactiflora. International Journal of Molecular Sciences. 20. 5953. 10.3390/ijms20235953.

Wang XY, Song J, Xing JH, Liang JF, and Ke BY. 2020a. Genome-Wide Identification and Expression Profile Analysis of WRKY Family Genes in Teak (Tectona Grandis). 10.21203/rs.3.rs-108100/v1.

Wang Y, Tang H, DeBarry JD, Tan X, Li J, Wang X, Lee Th, Jin H, Marler B, Guo H, Kissinger JC, and Paterson AH. 2012. MCScanX: a toolkit for detection and evolutionary analysis of gene synteny and collinearity. Nucleic Acids Research 40:e49-e49. 10.1093/nar/gkr1293.

Wei Y, Liu W, Hu W, Yan Y, and Shi H. 2019. The chaperone MeHSP90 recruits MeWRKY20 and MeCatalase1 to regulate drought stress resistance in cassava. New Phytologist. 10.1111/nph.16346.

Wei Y, Shi H, Xia Z, Tie W, Ding Z, Yan Y, Wang W, Hu W, and Li K. 2016. Genome-Wide Identification and Expression Analysis of the WRKY Gene Family in Cassava. Front Plant Sci 7:25. 10.3389/fpls.2016.00025.

Wilkins PW, and Humphreys MO. 2003. Progress in breeding perennial forage grasses for temperate agriculture. The Journal of Agricultural Science 140:129-150. $10.1017 / \mathrm{s} 0021859603003058$.

Wu GQ, Li ZQ, Cao H, and Wang JL. 2019. Distributed under Creative Commons CC-BY 4.0 Genome-wide identification and expression analysis of the WRKY genes in sugar beet (Beta vulgaris L.) under alkaline stress. PeerJ. 7. 10.7717/peerj.7817.

Xiang X, Chen J, Xu WX, Qiu JR, Song L, Wang JT, Tang R, Chen D, Jiang CZ, and Huang Z. 2021. Dehydration-Induced WRKY Transcriptional Factor MfWRKY70 of Myrothamnus flabellifolia Enhanced Drought and Salinity Tolerance in Arabidopsis. Biomolecules 11(2):327. 10.3390/biom11020327.

Xie W, Bushman BS, Ma Y, West MS, Robins JG, Michaels L, Jensen KB, Zhang X, Casler MD, and Stratton SD. 2014. Genetic diversity and variation in North American orchardgrass (Dactylis glomerataL.) cultivars and breeding lines. Grassland Science 60:185-193. 10.1111/grs.12058.

Yan H, Li MZ, Xiong Y, Wu JM, and Ma G. 2019. Genome-Wide Characterization, Expression Profile Analysis of WRKY Family Genes in Santalum album and Functional Identification of Their Role in Abiotic Stress. International Journal of Molecular Sciences. 20. 5676. 10.3390/ijms20225676. 
Yang XY, Zhou Z, Fu M, Han M, and Xu F. 2020. Transcriptome-wide identification of WRKY family genes and their expression profiling toward salicylic acid in Camellia japonica. Plant Signaling \& Behavior, 16(1), 1844508. 10.1080/15592324.2020.1844508.

Yu D, Chen C, and Chen Z. 2001. Evidence for an important role of WRKY DNA binding proteins in the regulation of NPR1 gene expression. The Plant cell 13:1527-1540. $10.1105 /$ tpc. 010115 .

Zandalinas SI, Mittler R, Balfagon D, Arbona V, and Gomez-Cadenas A. 2018. Plant adaptations to the combination of drought and high temperatures. Physiol Plant 162:2-12. 10.1111/ppl.12540.

Zeng B, Zhang Y, Zhang A, Qiao D, Ren J, Li M, Cai K, Zhang J, and Huang L. 2020. Transcriptome profiling of two Dactylis glomerata L. cultivars with different tolerance in response to submergence stress. Phytochemistry 175:112378. 10.1016/j.phytochem.2020.112378.

Zhang R, Murat F, Pont C, Langin T, and Salse J. 2014. Paleo-evolutionary plasticity of plant disease resistance genes. BMC Genomics 15:187. 10.1186/1471-2164-15-187.

Zhang Y, and Wang L. 2005. The WRKY transcription factor superfamily: its origin in eukaryotes and expansion in plants. BMC evolutionary biology 5:1.

Zhou QY, Tian AG, Zou HF, Xie ZM, Lei G, Huang J, Wang CM, Wang HW, Zhang JS, and Chen SY. 2008. Soybean WRKY-type transcription factor genes, GmWRKY13, GmWRKY21, and GmWRKY54, confer differential tolerance to abiotic stresses in transgenic Arabidopsis plants. Plant Biotechnol $J$ 6:486-503. 10.1111/j.14677652.2008.00336.x.

Ziheng Y, and Rasmus N. 2000. Estimating synonymous and nonsynonymous substitution rates under realistic evolutionary models. Molecular biology and evolution 17:32-43. 


\section{Figure 1}

The location of the WRKY gene family on different orchardgrass chromosomes.

The chromosome number is indicated at the top of the figure.
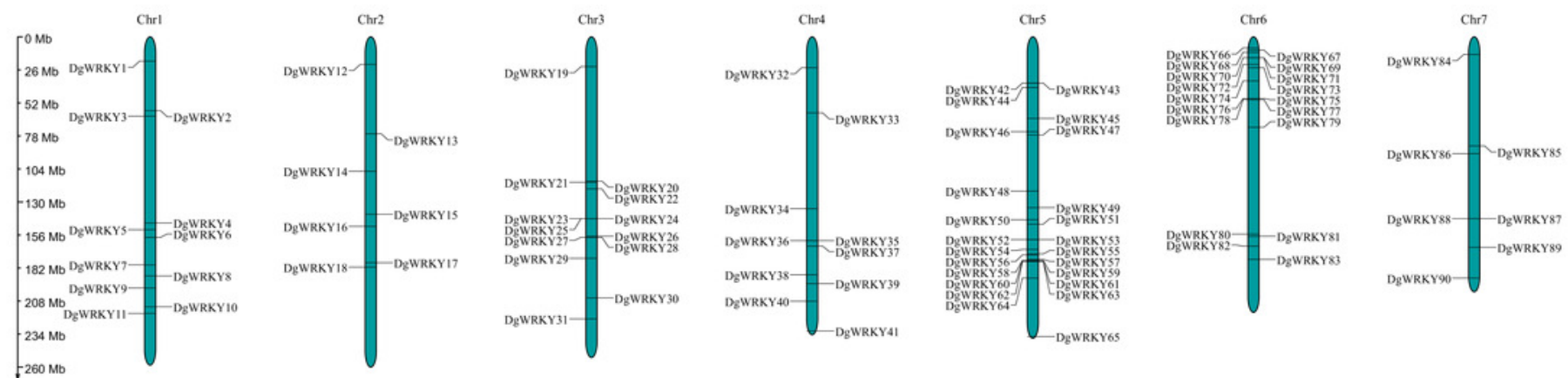
Figure 2

Phylogenetic tree obtained for the WRKY TF family members in, orchardgrass, wheat, and Arabidopsis.

Different colors represent different sub-classes in the WRKY gene family.

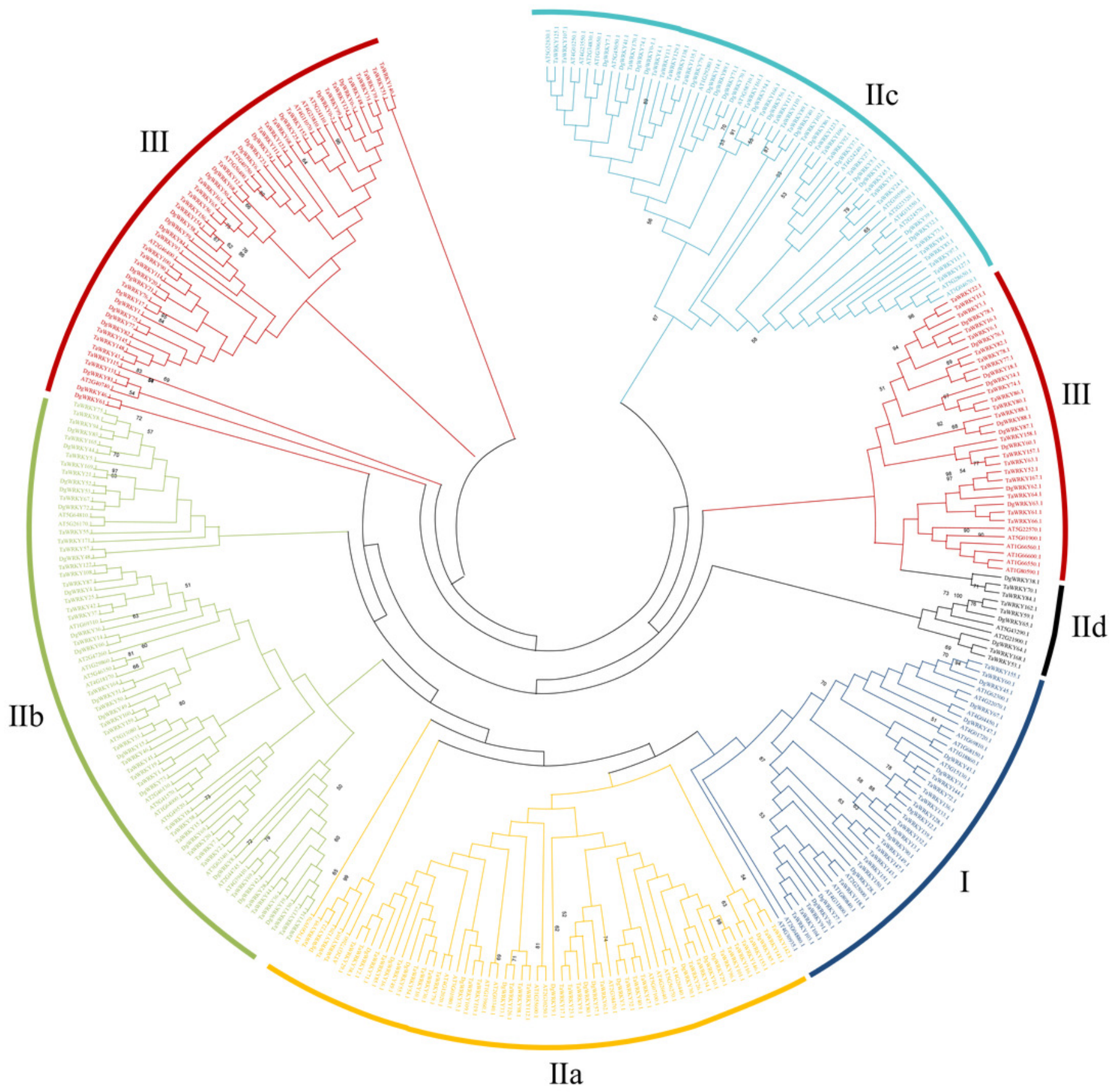




\section{Figure 3}

Multiple sequence alignments of the WRKY domains of from DgWRKYs.

The group name is indicated at the left of the figure. $-\mathrm{N}$ represents the N-terminal WRKY domains, $-\mathrm{C}$ represents the $\mathrm{C}$-terminal WRKY domains. (A) Multiple sequence alignments of the group I N-terminal. (B) Multiple sequence alignments of the group I C-terminal. (C) Multiple sequence alignments of the group Ila. (D) Multiple sequence alignments of the group IIb. (E) Multiple sequence alignments of the group IIc. (F) Multiple sequence alignments of the group IId. (G) Multiple sequence alignments of the group III N-terminal. (H) Multiple sequence alignments of the group III C-terminal. 


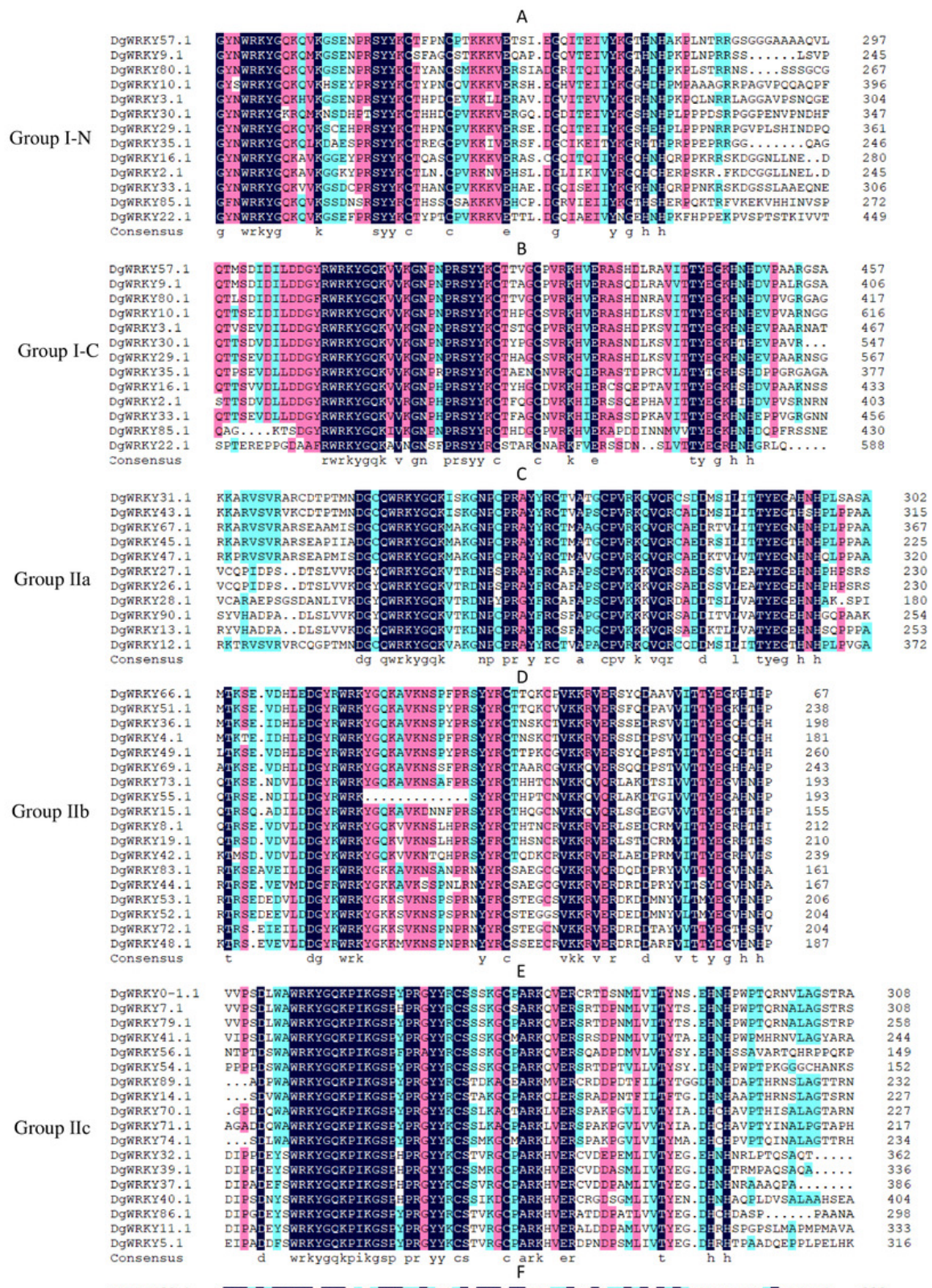

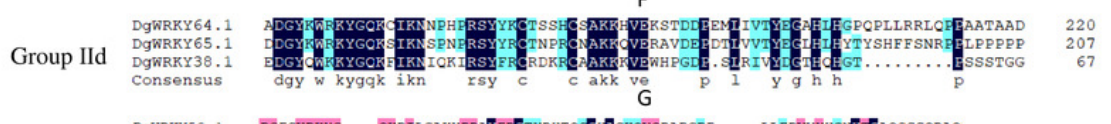

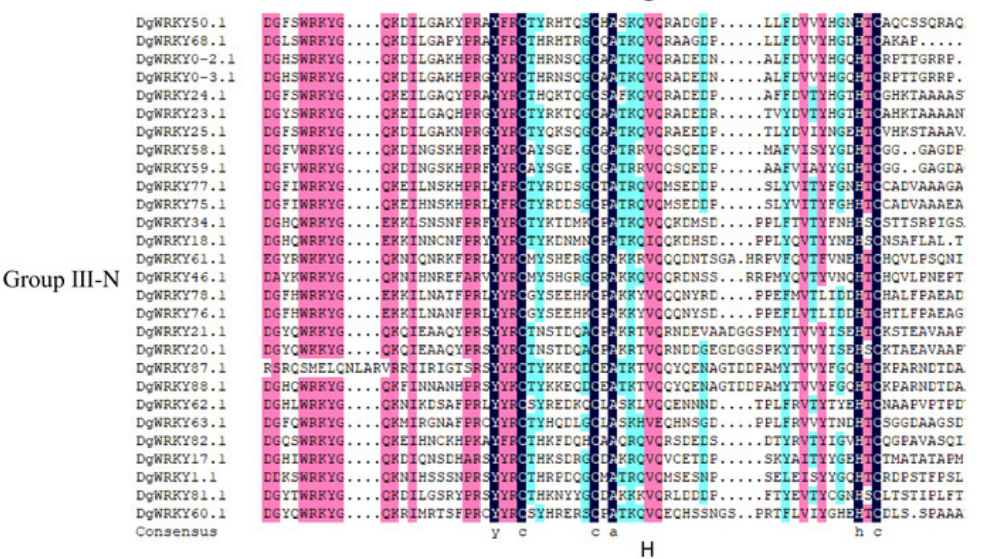

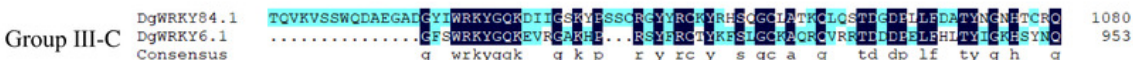




\section{Figure 4}

The phylogenetic tree, conserved motifs, and gene structure of orchardgrass WRKY family.

(A) Phylogenetic tree of WRKY proteins constructed by MEGA using the ML (Maximum

likelihood) method. (B) The motifs of WRKY protein are displayed in the figure. Different motifs are denoted by different colors numbered from motif 1-10 at the top right panel of the figure. The detailed information of the 10 motifs is listed in Figure S1. (C) The gene structure of 93 orchardgrass WRKY genes. The green boxes, yellow box, and full line represent CDS (Sequence coding for amino acids in protein), UTR (Untranslated region), and introns respectively. 
A
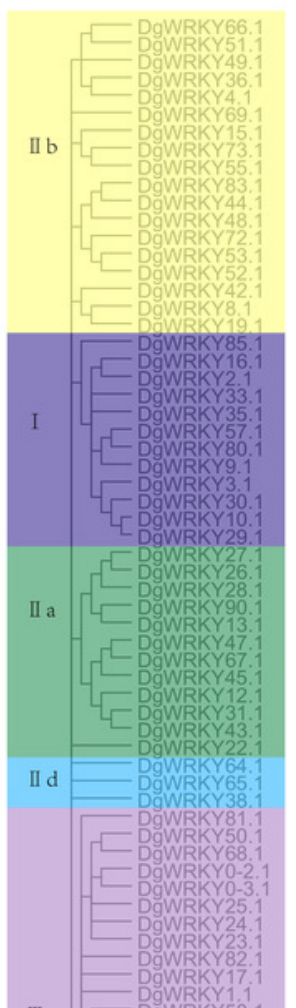

B

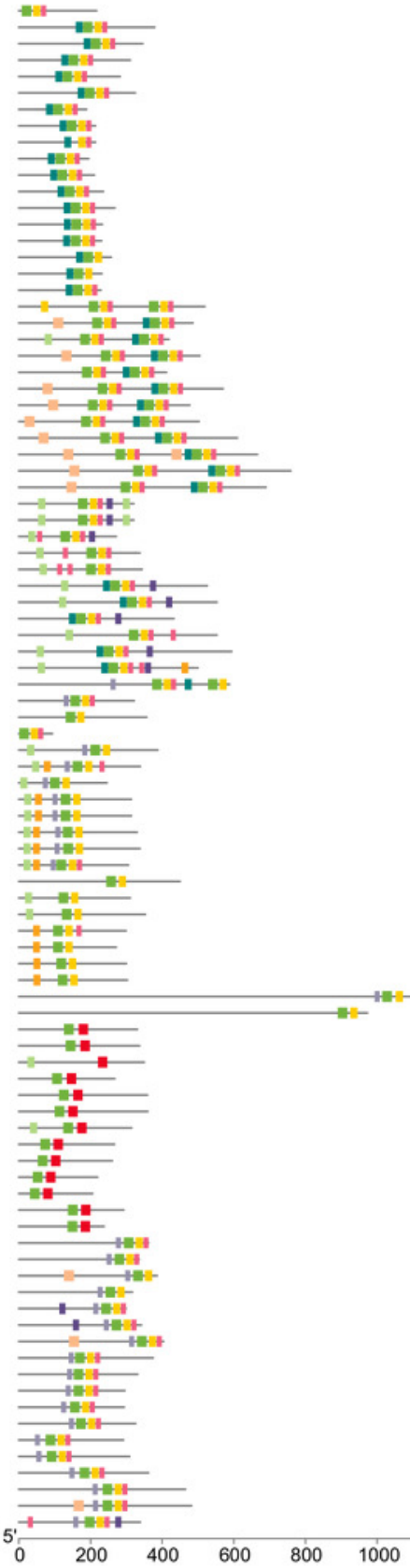

$\begin{array}{llllll} & 200 \quad 400 \quad 600 & 800 & 1000 & 1200 & 3\end{array}$
C

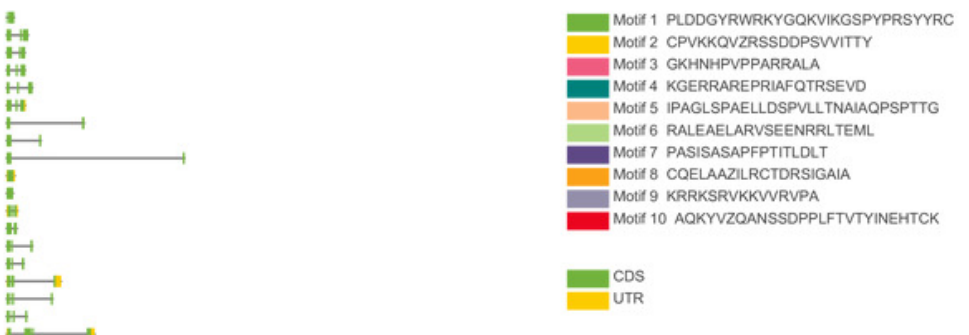

III

$\stackrel{\longrightarrow}{\longmapsto}$

$\rightarrow$

童

整山-

titin

H

$+$

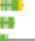

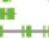

$\rightarrow$

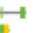

t

Hit

$+$

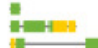

宙

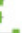

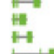

$\longleftrightarrow$

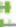

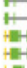

눈

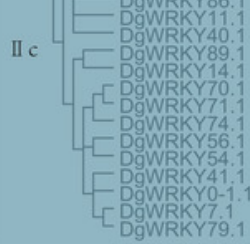

$\begin{array}{llllll}10000 & 20000 & 30000 & 40000 & 50000 & 60000\end{array}$ 
Figure 5

Genomic locations of tandem and segmentally duplicated gene pairs in the orchardgrass genome.

(A) Gray lines in the background indicate the synteny blocks within the whole orchardgrass genome, and red lines denote the segmental duplication of WRKY gene pairs. (B) Red lines denote the tandem duplication WRKY gene pairs, the gene name has been labeled.
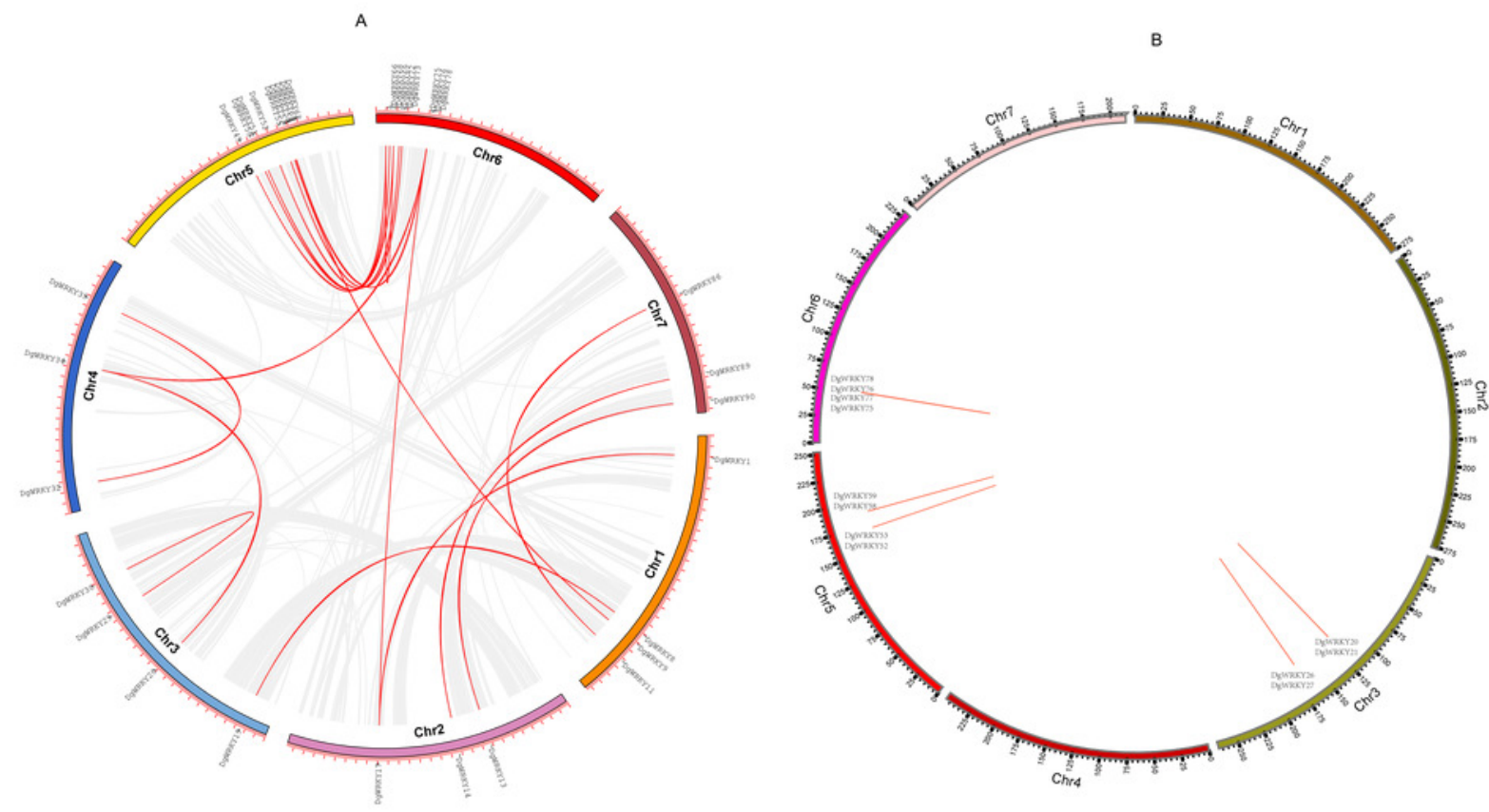
Figure 6

Expression of DgWRKY genes in root, stem, leaf, spike, flower tissues.

Blue to red color denotes low to high relative expression. The original expression values were normalized by Z-score normalization. 


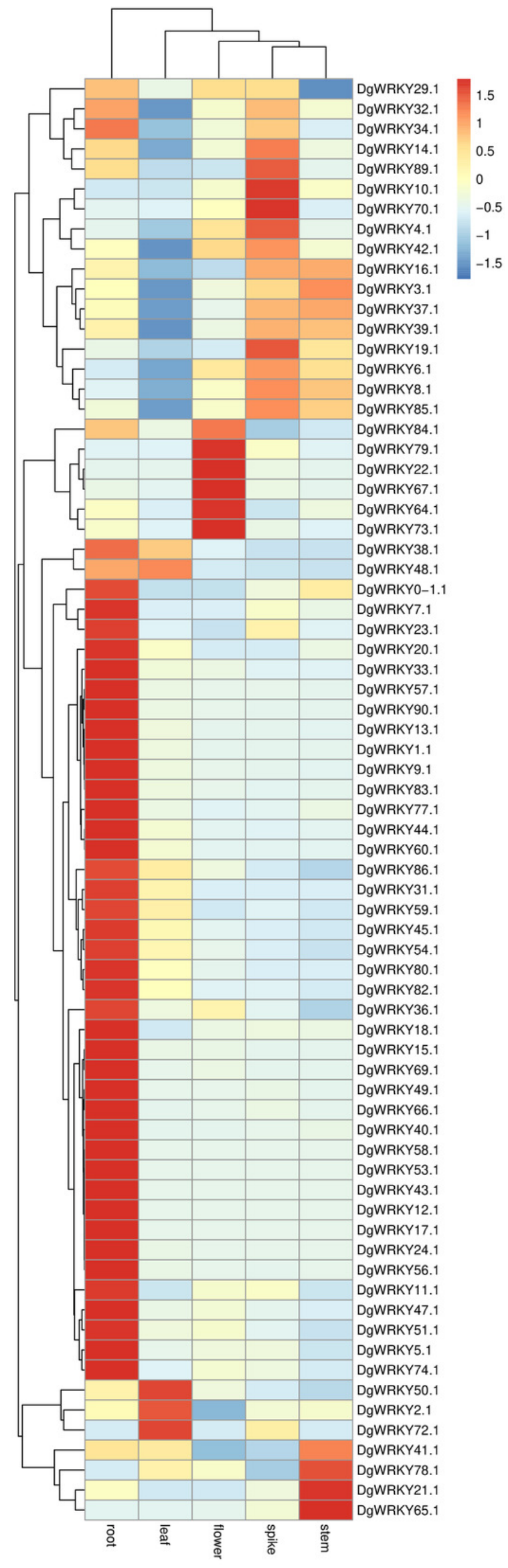




\section{Figure 7}

The number of the gene with the highest expression level in root, stem, leaf, spike, flower tissues.

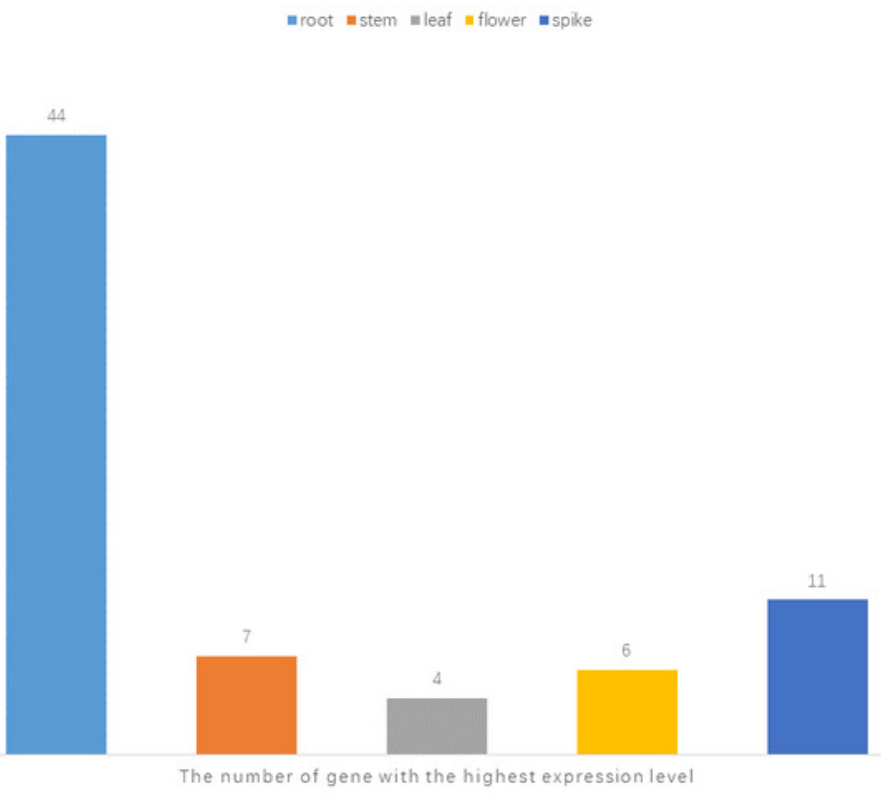




\section{Figure 8}

The expression profiles of WRKY genes (The sample names are shown at the bottom) in different abiotic stress of orchardgrass.

(A) DgWRKY expression patterns in 'BAOXING' (heat-resistant cultivar) and '01998' (heatsusceptible cultivar) under heat stress. (B) DgWRKY expression patterns in root and leaf under drought stress in 'BAOXING.' (C) DgWRKY expression patterns in 'DIANBEI' (submergence-tolerant cultivar) and 'ANBA' (submergence-susceptible cultivar) under submergence stress. Blue to red color denotes low to high relative expression. The original expression values were normalized by Z-score normalization. 
A

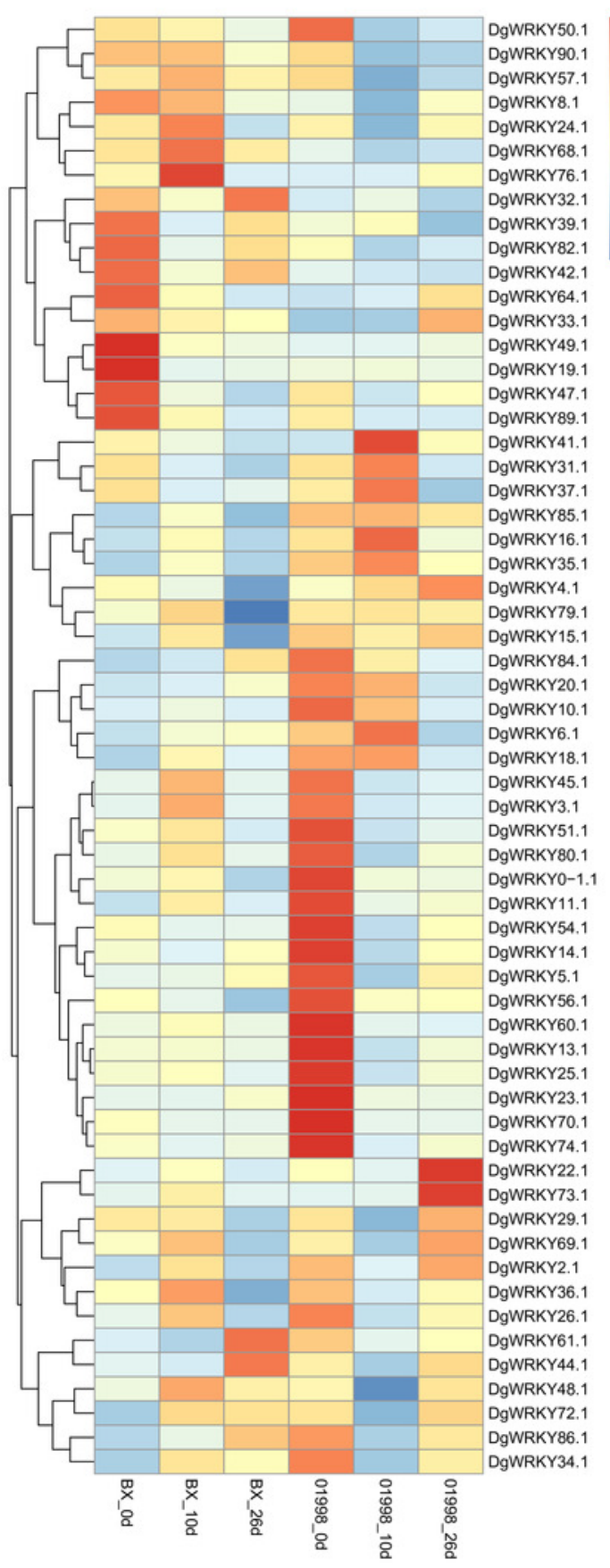

B

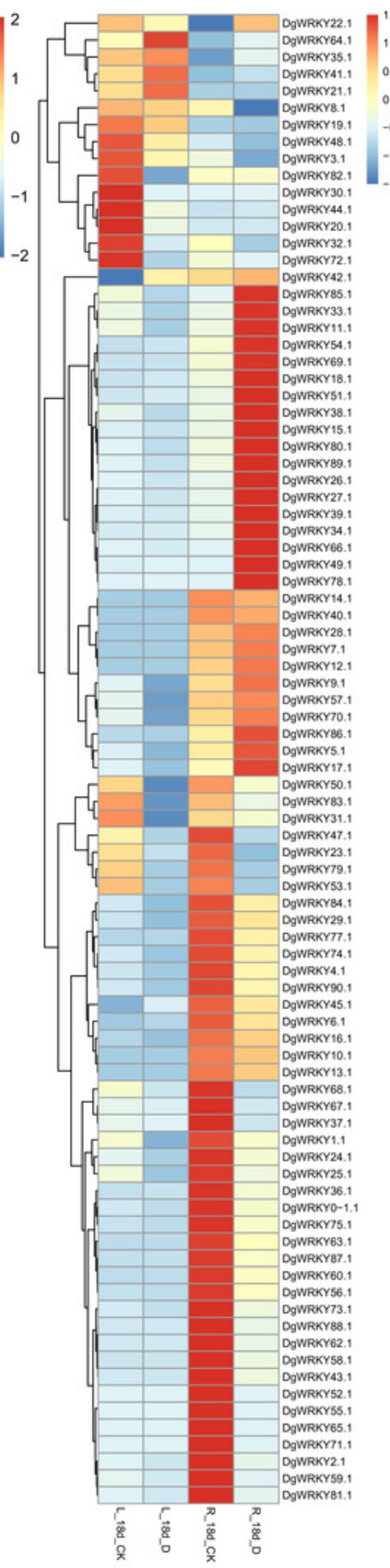

C

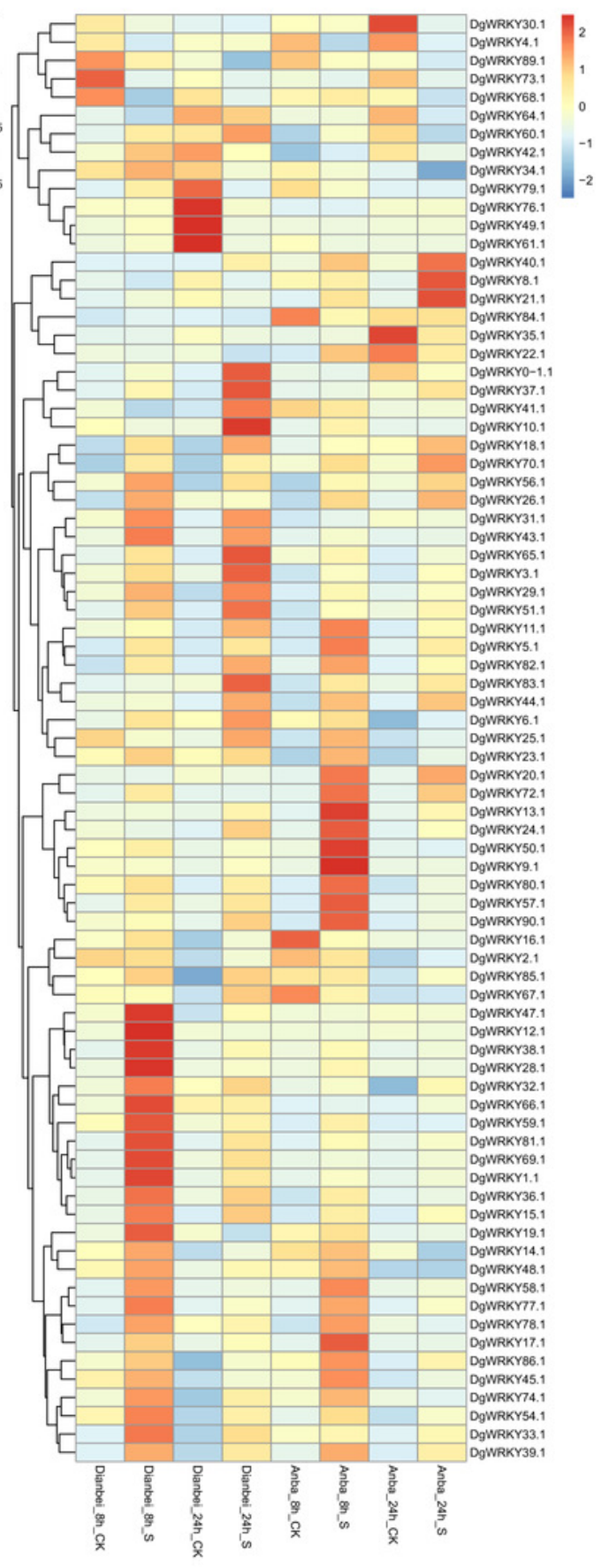




\section{Figure 9}

Venn diagram of different comparison groups.

(A) The comparison group of upregulated and downregulated genes in 'BAOXING' (heatresistant cultivar) and '01998' (heat-susceptible cultivar) at 10th day under heat stress. (B) The comparison group of upregulated and downregulated genes in 'BAOXING' and '01998' at 26th day under heat stress. (C) The comparison group of upregulated and downregulated genes in root and leaf of orchardgrass at 18th day under drought stress. (D) The comparison group of upregulated and downregulated genes in 'DIANBEI' (submergence-tolerant cultivar) and 'ANBA' (submergence-susceptible cultivar) at 8th hour under submergence stress. (E) The comparison group of upregulated and downregulated genes in 'DIANBEl' and 'ANBA' at 24th hour under submergence stress. (F) The comparison group of upregulated and downregulated genes in PI292589 (rust-susceptible line) and PI251814 (rust-resistant line) at 7 th day under rust stress. (G) The comparison group of upregulated and downregulated genes in PI292589 and PI251814 at 14th day under rust stress. (H) The comparison group of DEGs (differentially expressed genes) under heat, drought, submergence, and rust stress. 

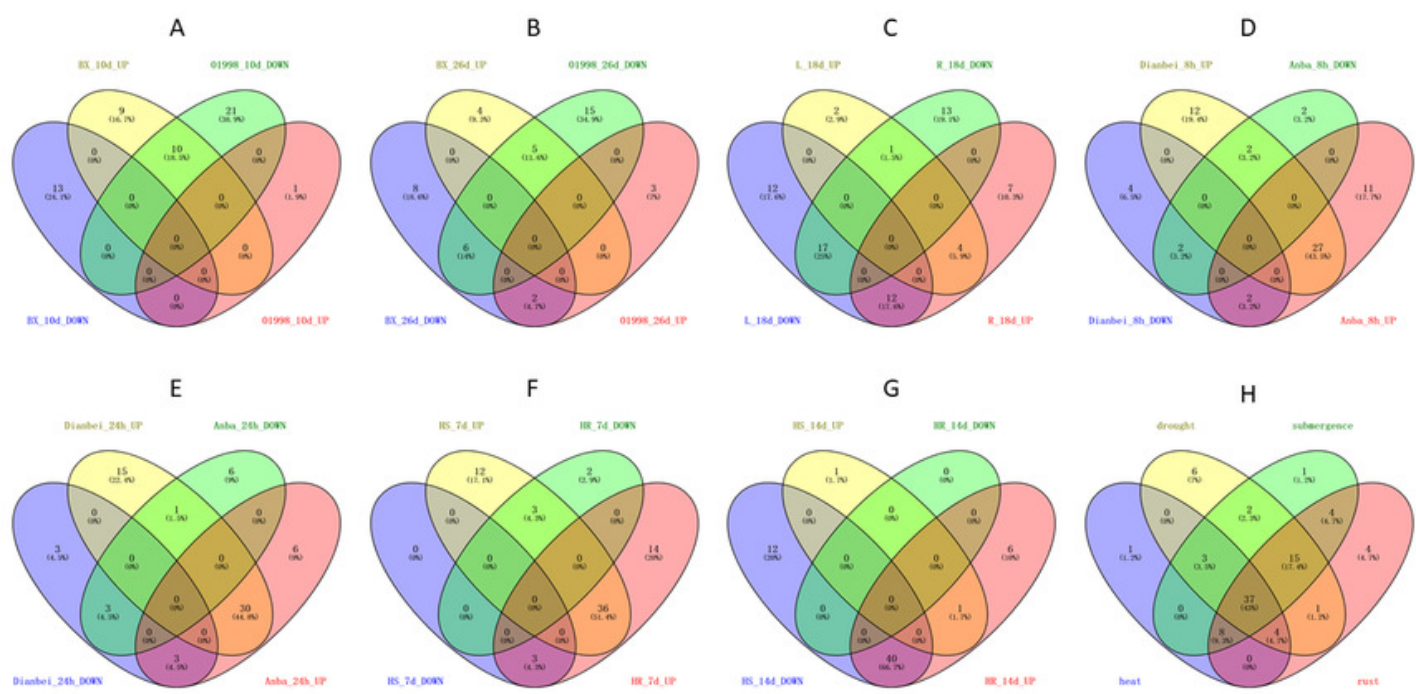


\section{Figure 10}

The expression profiles of WRKY genes in rust stress of orchardgrass, and box plot for significance test.

(A) The expression profiles of WRKY genes in rust stress of orchardgrass. The sample names are shown at the bottom. Blue to red color denotes low to high relative expression. The original expression values were normalized by Z-score normalization. (B) The box plot for significance test between PI292589 and PI251814. The more * symbols, the more significant the difference. (C) The box plot for significance test between HR_CK, HR_R, HS_CK, HS_R. HR stands for the highly rust-resistant PI251814 line. HS stands for the highly rust-susceptible PI292589 line. CK stands for no rust inoculation. R stands for rust inoculated. The more * symbols, the more significant the difference. 
A

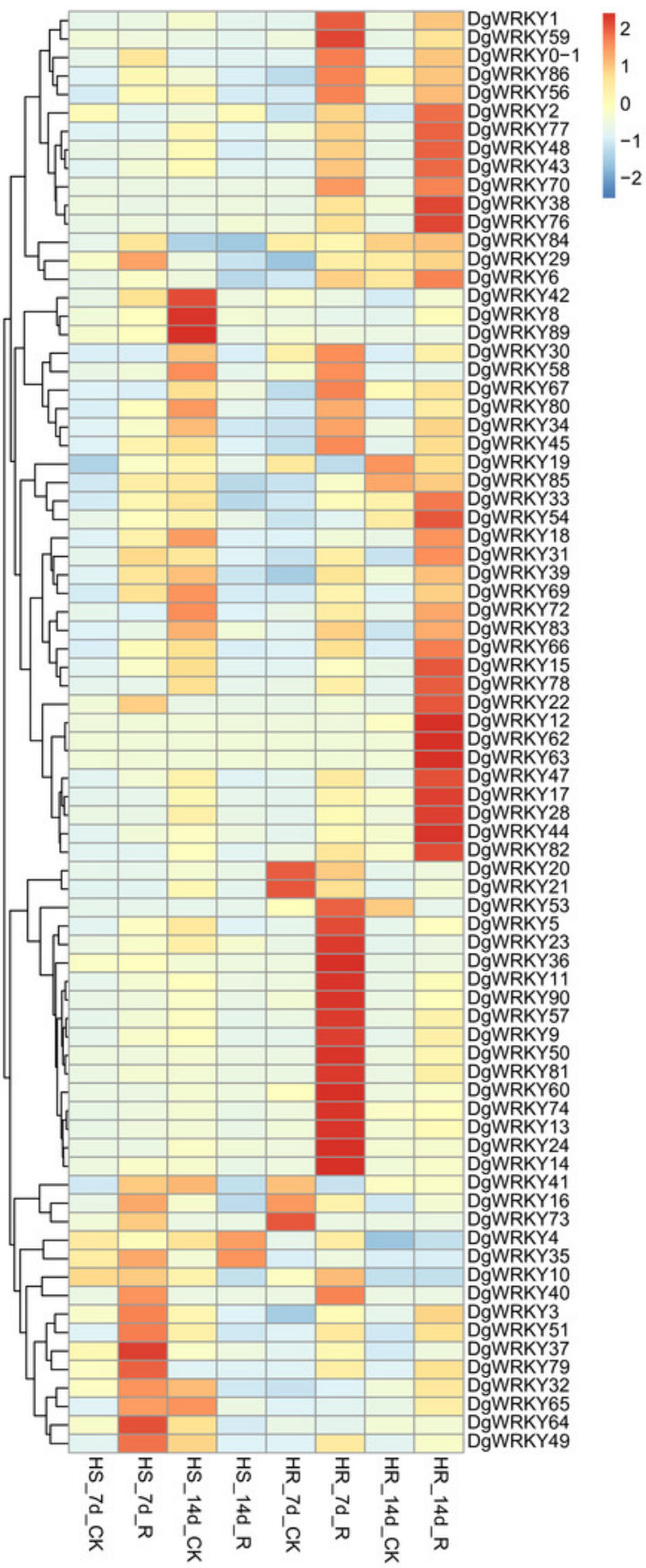

B

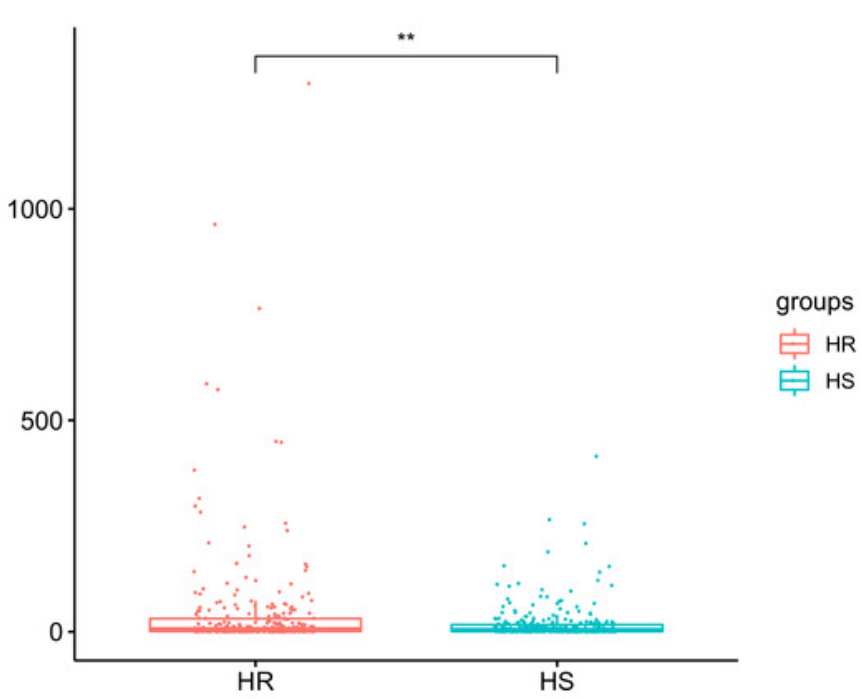

C

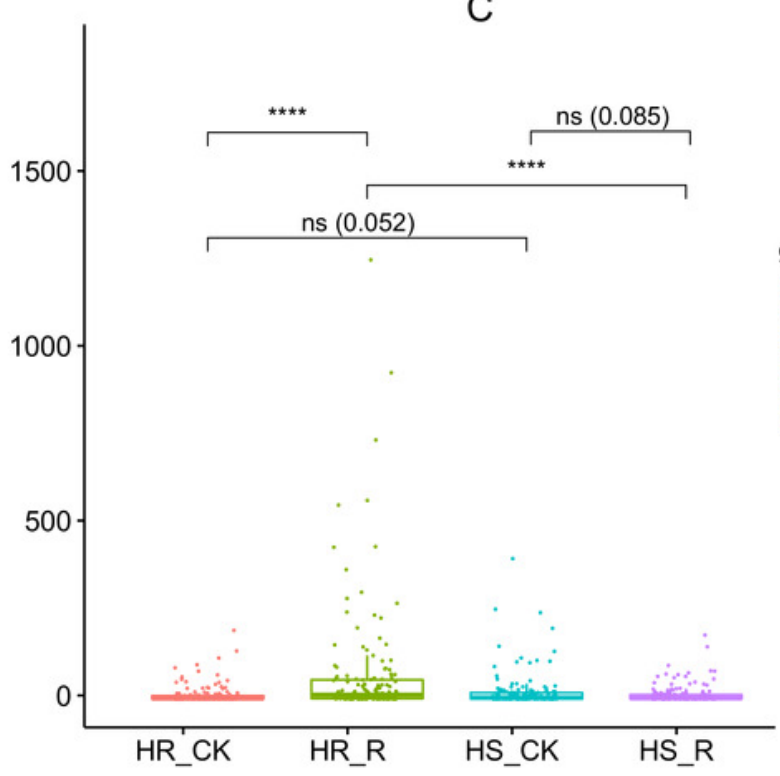




\section{Figure 11}

The number of DEGs (Differentially expressed genes) under heat, drought, submergence, and rust stress.

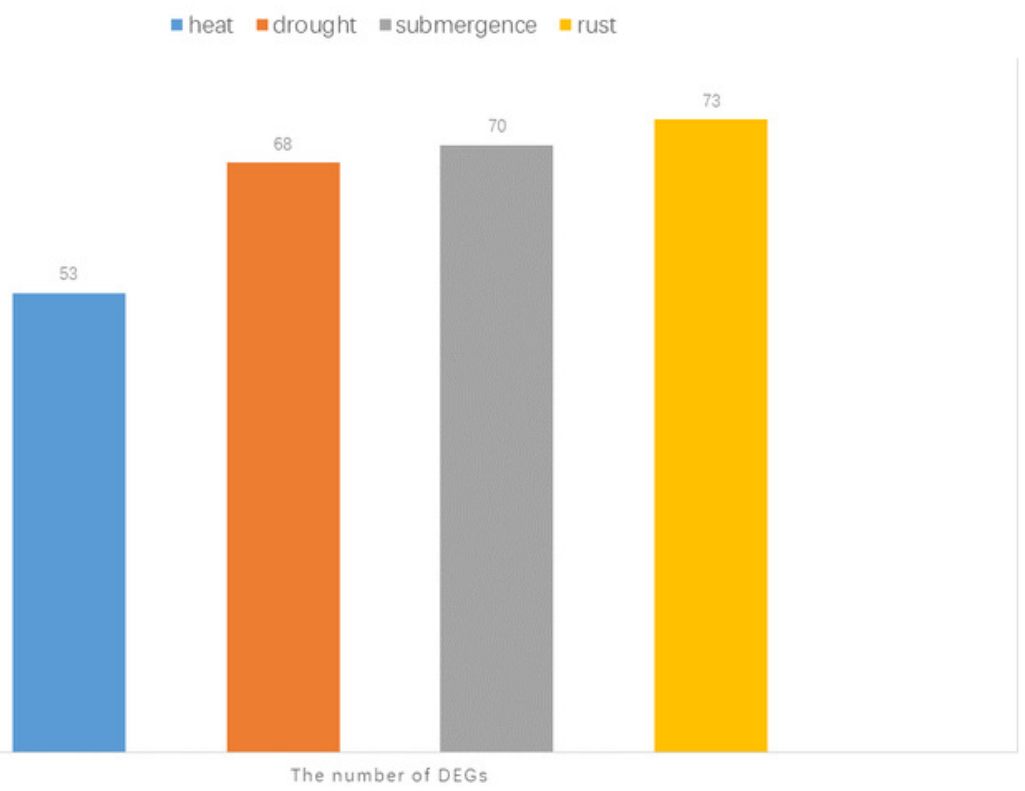


Figure 12

GO (Gene Ontology) categories of the target genes for 93 DgWRKYs.

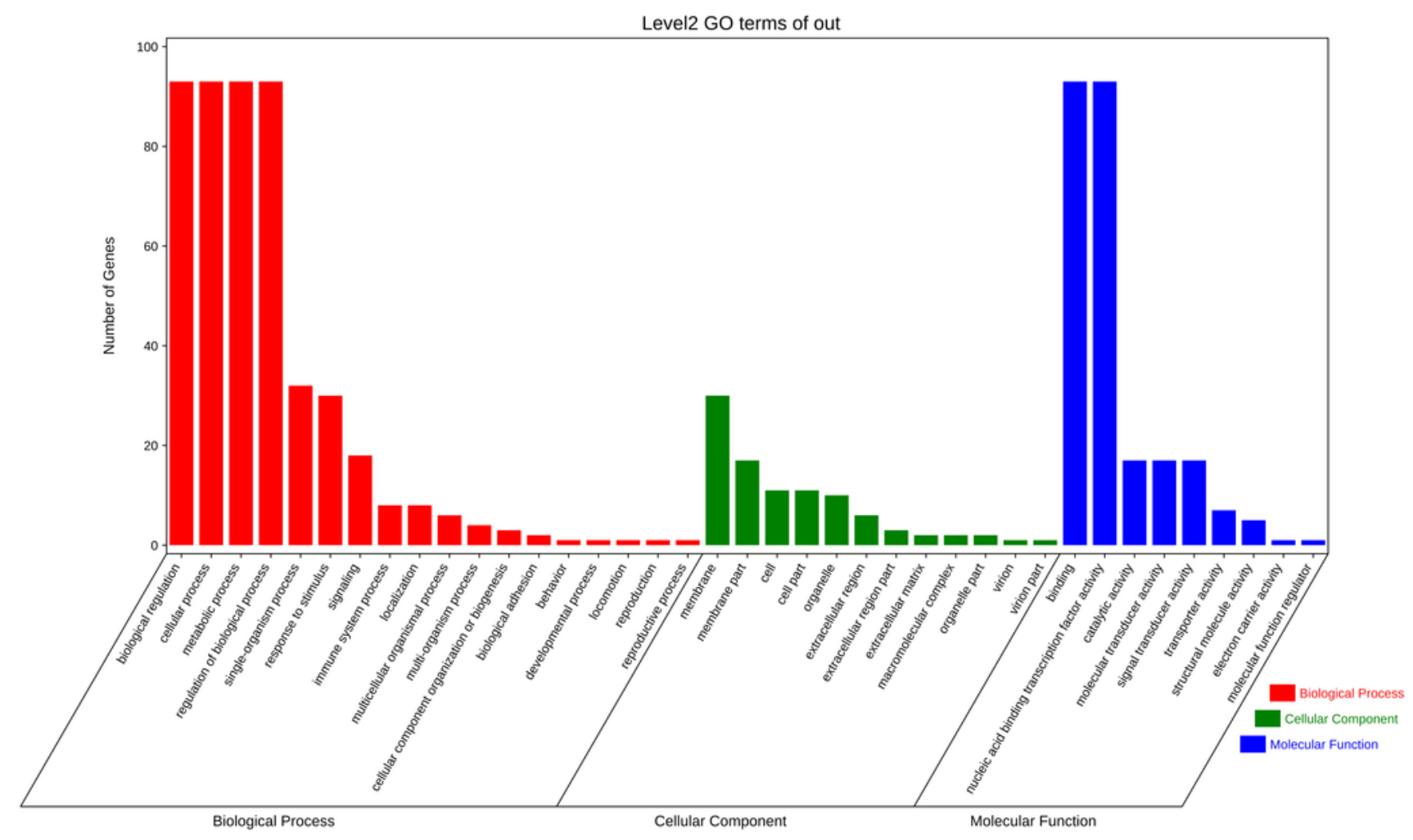


Figure 13

KEGG (Kyoto encyclopedia of genes and genomes) categories of the target genes for 93 DgWRKYs. 


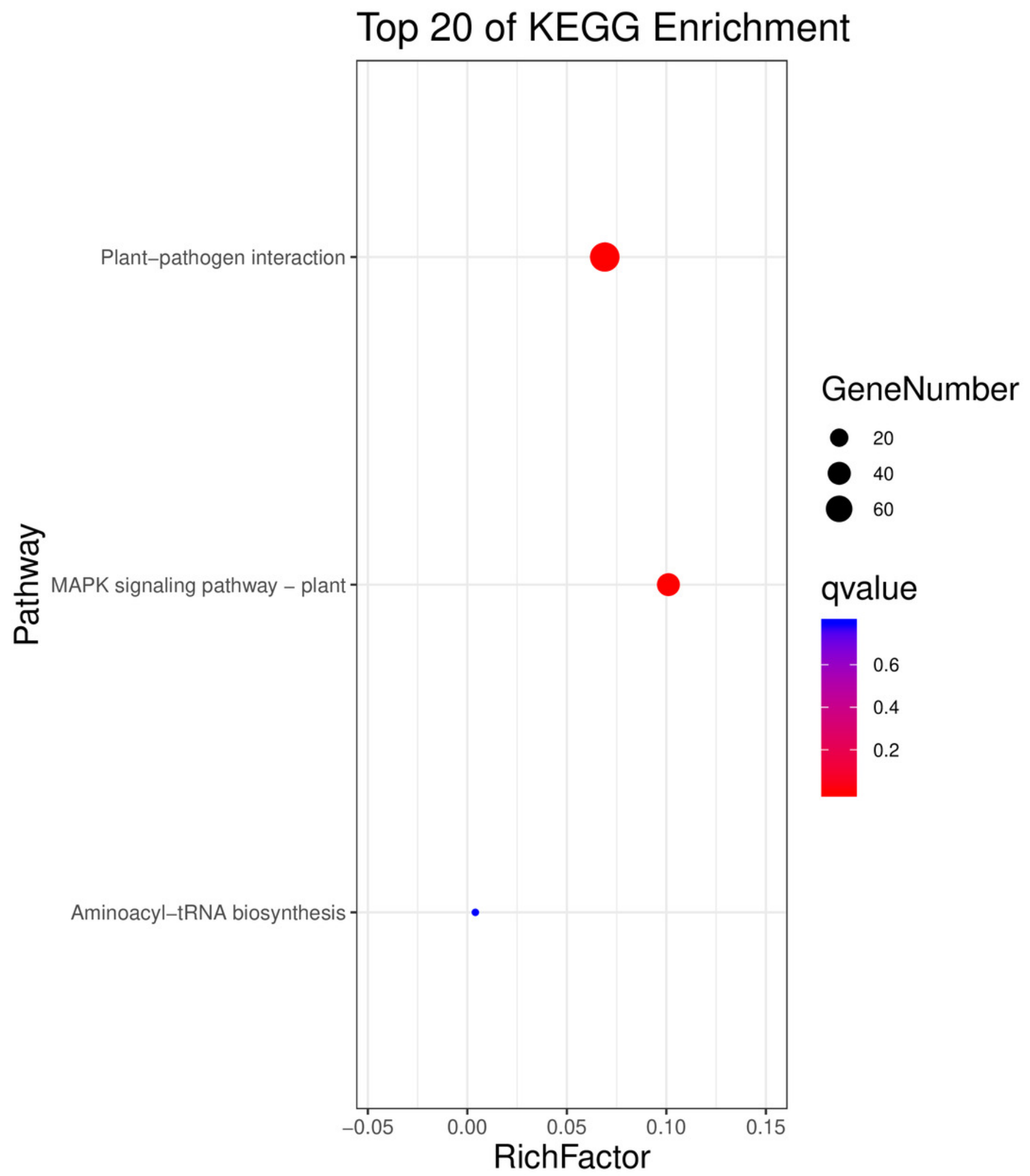


Figure 14

Predicted cis-elements in RGAs promoters.

Promoter sequences (-1500 bp) of 218 RGAs (just 154 have W-box) are analyzed by PlantCARE.
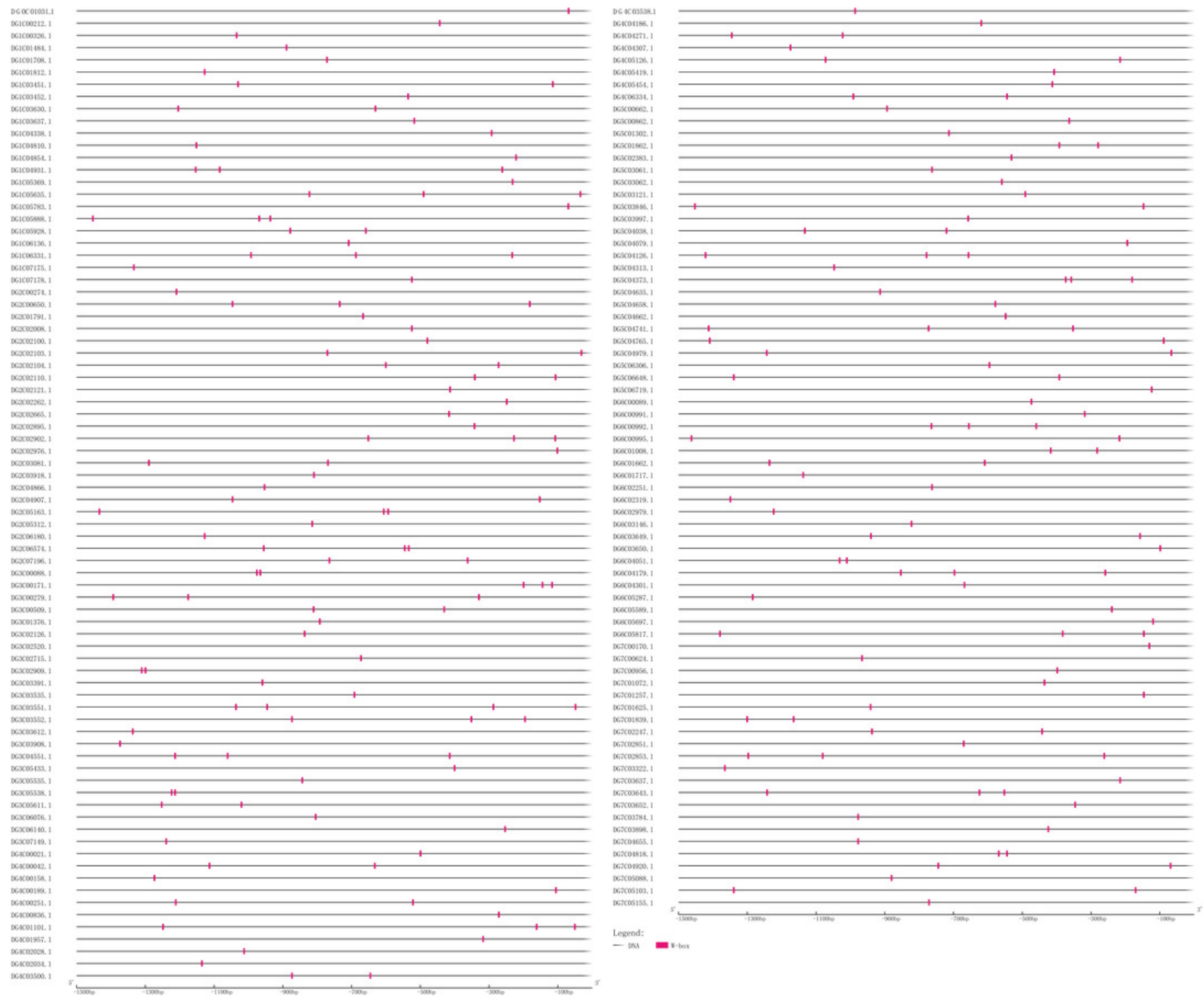
Figure 15

\section{Principal component analysis induced by rust.}

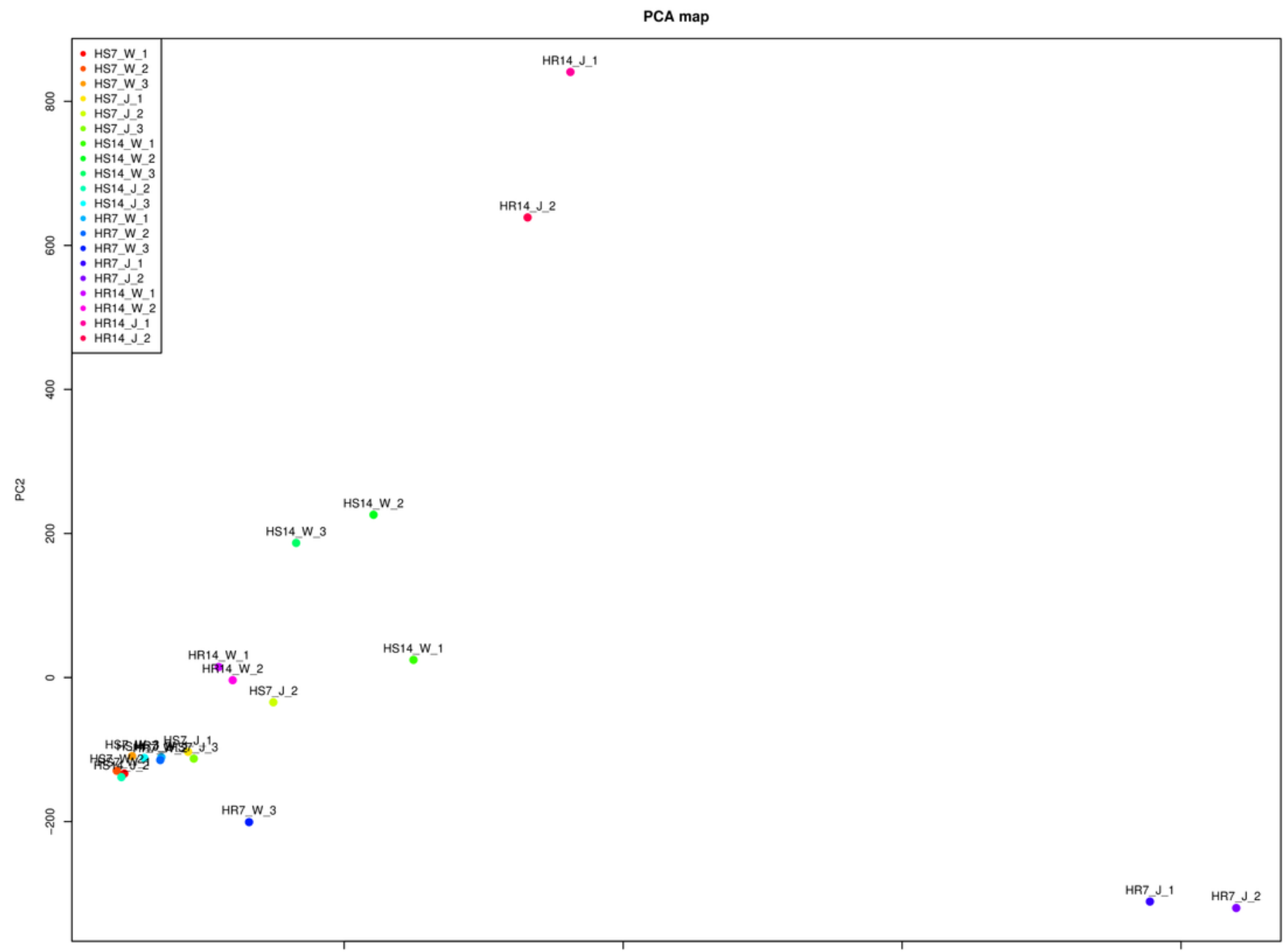


Figure 16

Heat map of correlation between modules and traits.

Red indicates a positive correlation, blue indicates a negative correlation, and the weighted correlation coefficient and $\mathrm{P}$ value are indicated in the box. 


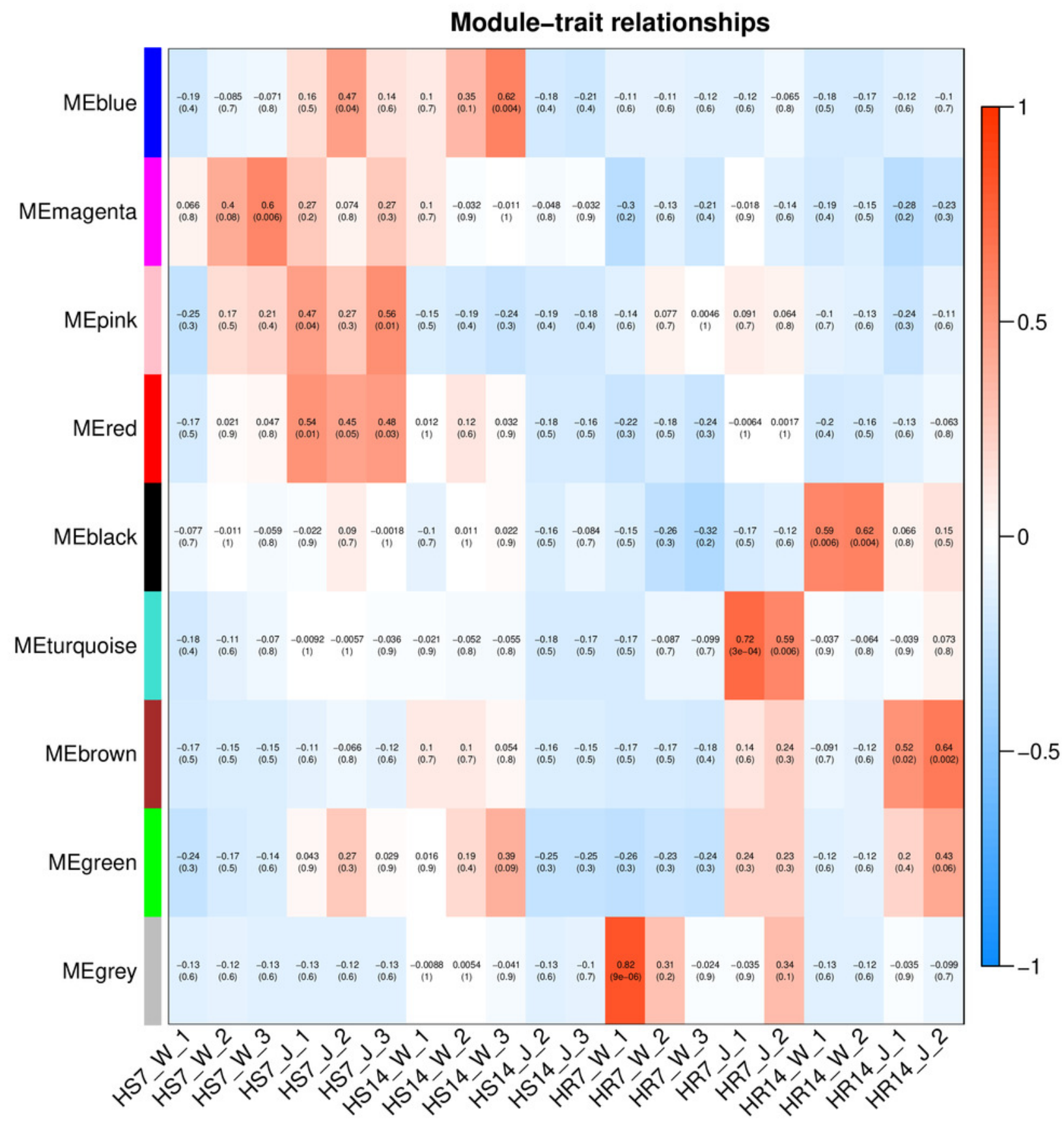




\section{Figure 17}

Interactions between 14 WRKYs and 5 RGAs.

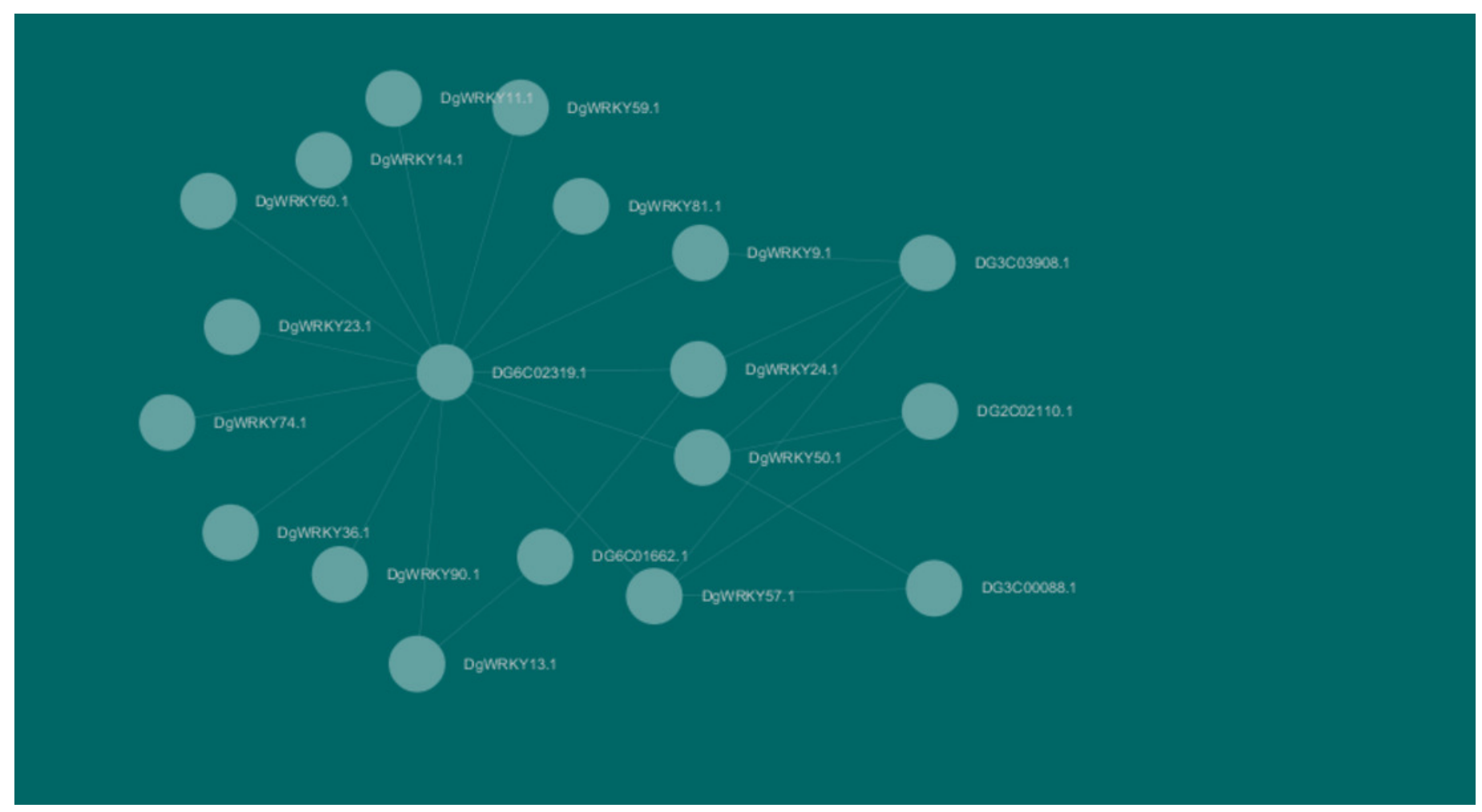




\section{Table $\mathbf{1}$ (on next page)}

DgWRKY proteins' physical and chemical properties and subcellular localization prediction.

MW, pl, GRAVY, Cyto, Nucl, Chlo, pero, and mito represent molecular weight, theoretical isoelectric points, grand average of hydropathicity cytoplasm, nucleus, chloroplast, peroxisome, and mitochondria, respectively. 


\begin{tabular}{|c|c|c|c|c|c|c|c|c|}
\hline Gene name & Group & Localization & length & MW & $\mathrm{pI}$ & Instability index & Aliphatic index & GRAVY \\
\hline DgWRKY10 & I & nucl & 759 & 81174.33 & 6.09 & 51.60 & 58.05 & -0.630 \\
\hline DgWRKY29 & I & nucl & 690 & 74783.88 & 6.10 & 58.05 & 50.78 & -0.742 \\
\hline DgWRKY30 & I & nucl & 666 & 72750.32 & 6.37 & 52.11 & 66.76 & -0.599 \\
\hline DgWRKY3 & I & nucl & 610 & 65850.26 & 6.56 & 56.53 & 52.64 & -0.782 \\
\hline DgWRKY22 & I & nucl & 588 & 64690.50 & 7.31 & 47.31 & 73.49 & -0.501 \\
\hline DgWRKY57 & I & nucl & 570 & 61042.40 & 6.33 & 58.08 & 39.51 & -0.842 \\
\hline DgWRKY85 & I & chlo & 519 & 56871.93 & 8.98 & 50.44 & 61.10 & -0.852 \\
\hline DgWRKY33 & I & nucl & 505 & 53501.74 & 8.59 & 63.27 & 53.21 & -0.823 \\
\hline DgWRKY9 & I & nucl & 503 & 53911.84 & 5.81 & 59.34 & 40.66 & -0.782 \\
\hline DgWRKY16 & I & nucl & 486 & 51550.55 & 8.73 & 60.52 & 55.76 & -0.741 \\
\hline DgWRKY80 & I & nucl & 477 & 50836.97 & 8.33 & 48.03 & 54.51 & -0.717 \\
\hline DgWRKY2 & I & nucl & 419 & 45348.61 & 5.96 & 48.94 & 71.03 & -0.568 \\
\hline DgWRKY35 & I & nucl & 412 & 44569.95 & 6.97 & 61.23 & 46.53 & -0.970 \\
\hline DgWRKY45 & II a & nucl & 866 & 90306.68 & 7.97 & 51.32 & 56.79 & -0.502 \\
\hline DgWRKY31 & II a & nucl & 594 & 62057.86 & 8.93 & 44.26 & 60.56 & -0.449 \\
\hline DgWRKY12 & II a & nucl & 553 & 58281.66 & 5.11 & 51.23 & 55.70 & -0.755 \\
\hline DgWRKY67 & II a & chlo & 553 & 57730.58 & 6.65 & 6.65 & 65.79 & -0.416 \\
\hline DgWRKY47 & I $\mathrm{a}$ & nucl & 526 & 56343.84 & 7.38 & 53.54 & 59.13 & -0.624 \\
\hline DgWRKY43 & II a & nucl & 500 & 52272.58 & 7.22 & 52.80 & 66.54 & -0.389 \\
\hline DgWRKY13 & II a & nucl & 344 & 37356.00 & 7.74 & 53.82 & 66.48 & -0.668 \\
\hline DgWRKY90 & II a & nucl & 338 & 36482.79 & 9.13 & 51.49 & 64.47 & -0.679 \\
\hline DgWRKY27 & I $\mathrm{a}$ & nucl & 321 & 34562.87 & 6.47 & 56.48 & 60.25 & -0.593 \\
\hline DgWRKY26 & II a & nucl & 321 & 34562.87 & 6.47 & 56.48 & 60.25 & -0.593 \\
\hline DgWRKY28 & II a & nucl & 272 & 29343.17 & 6.60 & 48.49 & 77.87 & -0.342 \\
\hline DgWRKY51 & II $\mathrm{b}$ & nucl & 379 & 39772.32 & 8.54 & 46.83 & 54.72 & -0.610 \\
\hline DgWRKY49 & $\Pi \mathrm{b}$ & nucl & 346 & 37879.06 & 6.67 & 63.51 & 50.20 & -0.845 \\
\hline DgWRKY69 & $\Pi \mathrm{b}$ & nucl & 325 & 34783.42 & 6.92 & 57.52 & 51.78 & -0.810 \\
\hline DgWRKY36 & $\Pi \mathrm{b}$ & nucl & 311 & 32487.14 & 8.36 & 59.73 & 55.59 & -0.463 \\
\hline DgWRKY4 & $\Pi \mathrm{b}$ & nucl & 283 & 30016.45 & 6.04 & 44.73 & 58.80 & -0.490 \\
\hline DgWRKY72 & II b & nucl & 268 & 28923.51 & 9.85 & 54.48 & 67.87 & -0.461 \\
\hline DgWRKY42 & II $\mathrm{b}$ & nucl & 258 & 27837.28 & 9.72 & 49.03 & 48.91 & -0.721 \\
\hline DgWRKY48 & $\Pi \mathrm{b}$ & cyto & 236 & 25755.53 & 5.97 & 55.33 & 55.47 & -0.644 \\
\hline DgWRKY53 & $\Pi \mathrm{b}$ & chlo & 233 & 24697.45 & 8.71 & 57.54 & 55.79 & -0.530 \\
\hline DgWRKY8 & $\Pi \mathrm{b}$ & nucl & 232 & 25259.58 & 8.55 & 38.58 & 62.67 & -0.449 \\
\hline DgWRKY52 & $\Pi \mathrm{b}$ & chlo & 231 & 24452.04 & 7.01 & 63.53 & 53.33 & -0.544 \\
\hline DgWRKY19 & $\Pi \mathrm{l} \mathrm{b}$ & nucl & 229 & 25943.34 & 8.79 & 51.93 & 51.05 & -0.641 \\
\hline DgWRKY66 & II b & nucl & 217 & 23544.33 & 9.58 & 59.34 & 52.44 & -0.653 \\
\hline DgWRKY55 & $\Pi \mathrm{b}$ & nucl & 214 & 23894.85 & 5.66 & 39.96 & 62.94 & -0.654 \\
\hline DgWRKY73 & $\Pi \mathrm{b}$ & nucl & 214 & 23315.11 & 6.65 & 42.03 & 71.59 & -0.593 \\
\hline DgWRKY44 & II b & nucl & 211 & 22099.16 & 8.37 & 44.98 & 49.34 & -0.505 \\
\hline
\end{tabular}




\begin{tabular}{|c|c|c|c|c|c|c|c|c|}
\hline DgWRKY83 & $\Pi \mathrm{b}$ & nucl & 195 & 21231.25 & 7.05 & 57.37 & 47.18 & -0.730 \\
\hline DgWRKY15 & II b & nucl & 189 & 21447.50 & 8.39 & 46.42 & 43.28 & -1.025 \\
\hline DgWRKY7 & II $\mathrm{c}$ & nucl & 482 & 51349.19 & 6.13 & 49.38 & 48.69 & -0.645 \\
\hline DgWRKY0-1 & II c & nucl & 465 & 49015.58 & 6.13 & 48.05 & 53.68 & -0.496 \\
\hline DgWRKY40 & $\Pi \mathrm{c}$ & nucl & 404 & 43627.13 & 9.37 & 60.10 & 62.87 & -0.582 \\
\hline DgWRKY37 & $\Pi \mathrm{c}$ & nucl & 386 & 41546.18 & 10.11 & 55.96 & 65.00 & -0.578 \\
\hline DgWRKY89 & II $\mathrm{c}$ & nucl & 375 & 39789.76 & 4.91 & 65.70 & 50.29 & -0.761 \\
\hline DgWRKY41 & II $\mathrm{c}$ & nucl & 362 & 39076.64 & 6.43 & 47.28 & 62.82 & -0.540 \\
\hline DgWRKY32 & $\Pi \mathrm{c}$ & nucl & 362 & 38951.35 & 10.01 & 55.14 & 60.39 & -0.561 \\
\hline DgWRKY11 & II c & pero & 342 & 36213.18 & 9.83 & 49.99 & 67.13 & -0.427 \\
\hline DgWRKY79 & II c & nucl & 339 & 37029.67 & 6.19 & 50.30 & 65.63 & -0.569 \\
\hline DgWRKY39 & $\Pi \mathrm{c}$ & nucl & 336 & 36699.61 & 9.79 & 46.81 & 62.44 & -0.616 \\
\hline DgWRKY14 & $\Pi \mathrm{c}$ & nucl & 332 & 35413.52 & 6.08 & 64.66 & 53.61 & -0.640 \\
\hline DgWRKY74 & II $\mathrm{c}$ & nucl & 326 & 35117.48 & 6.46 & 52.00 & 60.25 & -0.609 \\
\hline DgWRKY5 & $\Pi \mathrm{c}$ & nucl & 317 & 33740.11 & 9.61 & 51.51 & 57.03 & -0.636 \\
\hline DgWRKY54 & II c & nucl & 309 & 33685.98 & 4.83 & 81.08 & 49.94 & -0.917 \\
\hline DgWRKY86 & $\Pi \mathrm{c}$ & nucl & 299 & 31307.43 & 10.05 & 54.79 & 64.78 & -0.454 \\
\hline DgWRKY70 & II c & nucl & 296 & 31660.20 & 5.84 & 55.63 & 63.01 & -0.525 \\
\hline DgWRKY71 & $\Pi \mathrm{c}$ & nucl & 294 & 31792.51 & 5.16 & 54.38 & 67.35 & -0.509 \\
\hline DgWRKY56 & II $\mathrm{c}$ & nucl & 292 & 31133.43 & 5.13 & 58.90 & 54.11 & -0.666 \\
\hline DgWRKY65 & $\Pi \mathrm{d}$ & nucl & 357 & 40728.89 & 9.98 & 73.05 & 47.90 & -0.959 \\
\hline DgWRKY64 & $\Pi \mathrm{d}$ & nucl & 322 & 34437.13 & 6.22 & 58.32 & 58.29 & -0.744 \\
\hline DgWRKY38 & $\Pi \mathrm{d}$ & nucl & 94 & 10639.82 & 9.33 & 36.45 & 41.49 & -1.180 \\
\hline DgWRKY84 & III & nucl & 1114 & 126056.21 & 8.49 & 46.14 & 89.43 & -0.274 \\
\hline DgWRKY6 & III & cyto & 973 & 111435.07 & 6.46 & 40.31 & 101.39 & -0.167 \\
\hline DgWRKY82 & III & chlo & 450 & 49425.21 & 9.12 & 52.21 & 63.60 & -0.578 \\
\hline DgWRKY81 & III & nucl & 388 & 41378.33 & 6.05 & 54.13 & 67.76 & -0.388 \\
\hline DgWRKY46 & III & nucl & 360 & 39955.21 & 8.29 & 62.40 & 70.72 & -0.453 \\
\hline DgWRKY61 & III & nucl & 359 & 39654.04 & 6.26 & 56.12 & 67.60 & -0.369 \\
\hline DgWRKY1 & III & nucl & 353 & 37047.26 & 8.92 & 62.13 & 53.43 & -0.442 \\
\hline DgWRKY87 & III & chlo & 350 & 38159.62 & 7.48 & 62.28 & 64.17 & -0.498 \\
\hline DgWRKY50 & III & nucl & 339 & 36124.25 & 6.70 & 48.39 & 61.15 & -0.442 \\
\hline DgWRKY24 & III & nucl & 338 & 36343.56 & 5.39 & 55.23 & 63.02 & -0.405 \\
\hline DgWRKY20 & III & nucl & 337 & 35423.42 & 6.60 & 51.34 & 63.23 & -0.379 \\
\hline DgWRKY21 & III & nucl & 331 & 35153.98 & 6.24 & 53.49 & 61.36 & -0.447 \\
\hline DgWRKY25 & III & nucl & 330 & 35600.91 & 6.66 & 57.29 & 62.67 & -0.436 \\
\hline DgWRKY60 & III & nucl & 315 & 33705.31 & 5.34 & 54.52 & 66.10 & -0.459 \\
\hline DgWRKY0-2 & III & nucl & 314 & 33810.94 & 6.09 & 54.55 & 72.42 & -0.361 \\
\hline DgWRKY0-3 & III & nucl & 314 & 33810.94 & 6.09 & 54.55 & 72.42 & -0.361 \\
\hline DgWRKY17 & III & nucl & 311 & 32769.88 & 5.79 & 56.51 & 57.46 & -0.539 \\
\hline DgWRKY23 & III & nucl & 306 & 33359.39 & 6.06 & 54.35 & 67.61 & -0.430 \\
\hline
\end{tabular}




\begin{tabular}{lllllllll} 
DgWRKY75 & III & nucl & 303 & 32361.68 & 5.25 & 49.32 & 65.54 & -0.457 \\
DgWRKY77 & III & nucl & 300 & 32191.45 & 5.10 & 52.75 & 62.30 & -0.468 \\
DgWRKY58 & III & nucl & 299 & 31712.53 & 5.31 & 54.30 & 67.66 & -0.305 \\
DgWRKY78 & III & nucl & 293 & 32354.14 & 6.52 & 70.16 & 61.71 & -0.668 \\
DgWRKY59 & III & nucl & 272 & 28658.00 & 7.00 & 55.22 & 64.38 & -0.324 \\
DgWRKY88 & III & chlo & 268 & 29925.58 & 8.70 & 49.93 & 64.48 & -0.651 \\
DgWRKY62 & III & nucl & 267 & 29921.99 & 5.84 & 61.26 & 44.27 & -0.870 \\
DgWRKY63 & III & nucl & 261 & 28685.06 & 6.54 & 48.53 & 63.22 & -0.546 \\
DgWRKY68 & III & mito & 246 & 26701.98 & 6.59 & 38.64 & 67.15 & -0.478 \\
DgWRKY76 & III & nucl & 238 & 26066.07 & 8.78 & 74.88 & 63.70 & -0.720 \\
DgWRKY34 & III & nucl & 220 & 24507.97 & 7.61 & 66.38 & 43.55 & -0.838 \\
DgWRKY18 & III & nucl & 206 & 22874.32 & 8.44 & 58.24 & 46.99 & -0.862 \\
\hline
\end{tabular}

1 
Table 2 (on next page)

The $\mathrm{K}_{\mathrm{a}} / \mathrm{K}_{\mathrm{s}}$ of 4 tandem repeat gene pairs. 


\begin{tabular}{lllll}
\hline Gene name & $\mathrm{Ka}$ & $\mathrm{Ks}$ & $\mathrm{Ka} / \mathrm{Ks}$ & Length \\
\hline DgWRKY52 \& DgWRKY53 & 0.050 & 0.149 & 0.337 & 687 \\
DgWRKY76 \& DgWRKY78 & 0.032 & 0.091 & 0.352 & 705 \\
DgWRKY75 \& DgWRKY77 & 0.052 & 0.149 & 0.346 & 894 \\
DgWRKY20 \& DgWRKY21 & 0.084 & 0.255 & 0.330 & 984 \\
\hline
\end{tabular}

1 
Table 3 (on next page)

Pearson correlation coefficient for pairs. 


\begin{tabular}{|c|c|c|c|}
\hline DgWRKYs & RGAs & cor & P-value \\
\hline DgWRKY11.1 & DG6C02319.1 & 0.965717222 & $5.48582 \mathrm{E}-12$ \\
\hline DgWRKY13.1 & DG6C01662.1 & 0.966808979 & 4.11599E-12 \\
\hline DgWRKY13.1 & DG6C02319.1 & 0.972198837 & $8.51905 \mathrm{E}-13$ \\
\hline DgWRKY14.1 & DG6C02319.1 & 0.962503072 & $1.21456 \mathrm{E}-11$ \\
\hline DgWRKY23.1 & DG6C02319.1 & 0.841006826 & $3.41018 \mathrm{E}-06$ \\
\hline DgWRKY24.1 & DG6C01662.1 & 0.930842849 & $2.6691 \mathrm{E}-09$ \\
\hline DgWRKY24.1 & DG6C02319.1 & 0.961464602 & $1.54719 \mathrm{E}-11$ \\
\hline DgWRKY24.1 & DG3C03908.1 & 0.964729314 & $7.05855 \mathrm{E}-12$ \\
\hline DgWRKY36.1 & DG6C02319.1 & 0.935563084 & $1.43759 \mathrm{E}-09$ \\
\hline DgWRKY50.1 & DG2C02110.1 & 0.895697806 & $9.45072 \mathrm{E}-08$ \\
\hline DgWRKY50.1 & DG6C02319.1 & 0.956800712 & $4.25282 \mathrm{E}-11$ \\
\hline DgWRKY50.1 & DG3C03908.1 & 0.96377025 & $8.95481 \mathrm{E}-12$ \\
\hline DgWRKY50.1 & DG3C00088.1 & 0.960773767 & $1.81091 \mathrm{E}-11$ \\
\hline DgWRKY57.1 & DG2C02110.1 & 0.901223329 & 5.91198E-08 \\
\hline DgWRKY57.1 & DG6C02319.1 & 0.953225134 & $8.58626 \mathrm{E}-11$ \\
\hline DgWRKY57.1 & DG3C03908.1 & 0.966609051 & $4.34141 \mathrm{E}-12$ \\
\hline DgWRKY57.1 & DG3C00088.1 & 0.949987128 & $1.54974 \mathrm{E}-10$ \\
\hline DgWRKY59.1 & DG6C02319.1 & 0.823333144 & $8.22376 \mathrm{E}-06$ \\
\hline DgWRKY60.1 & DG6C02319.1 & 0.901864922 & $5.58866 \mathrm{E}-08$ \\
\hline DgWRKY74.1 & DG6C02319.1 & 0.866684293 & 7.70972E-07 \\
\hline DgWRKY81.1 & DG6C02319.1 & 0.914834278 & $1.63815 \mathrm{E}-08$ \\
\hline DgWRKY9.1 & DG6C02319.1 & 0.939321965 & $8.48671 \mathrm{E}-10$ \\
\hline DgWRKY9.1 & DG3C03908.1 & 0.955631067 & $5.38563 \mathrm{E}-11$ \\
\hline DgWRKY90.1 & DG6C02319.1 & 0.871137929 & 5.77559E-07 \\
\hline
\end{tabular}

